\title{
Assessing the feasibility, acceptability, and costs of diagnosing HIV at birth in Lesotho and Rwanda
}

Michelle M. Gill

Heather Hoffman

Majoalane Mokone

Lynne Mofenson

Michel Tchuenche

See next page for additional authors

Follow this and additional works at: https://knowledgecommons.popcouncil.org/departments_sbsr-hiv

Part of the Demography, Population, and Ecology Commons, Family, Life Course, and Society Commons, International Public Health Commons, and the Maternal and Child Health Commons How does access to this work benefit you? Let us know!

\section{Recommended Citation}

Gill, Michelle M., Heather Hoffman, Majoalane Mokone, Lynne Mofenson, Michel Tchuenche, and Steven Forsythe. 2018. "Assessing the feasibility, acceptability, and costs of diagnosing HIV at birth in Lesotho and Rwanda," Project SOAR Final Report. Washington, DC: Population Council. 
Authors

Michelle M. Gill, Heather Hoffman, Majoalane Mokone, Lynne Mofenson, Michel Tchuenche, and Steven

Forsythe 


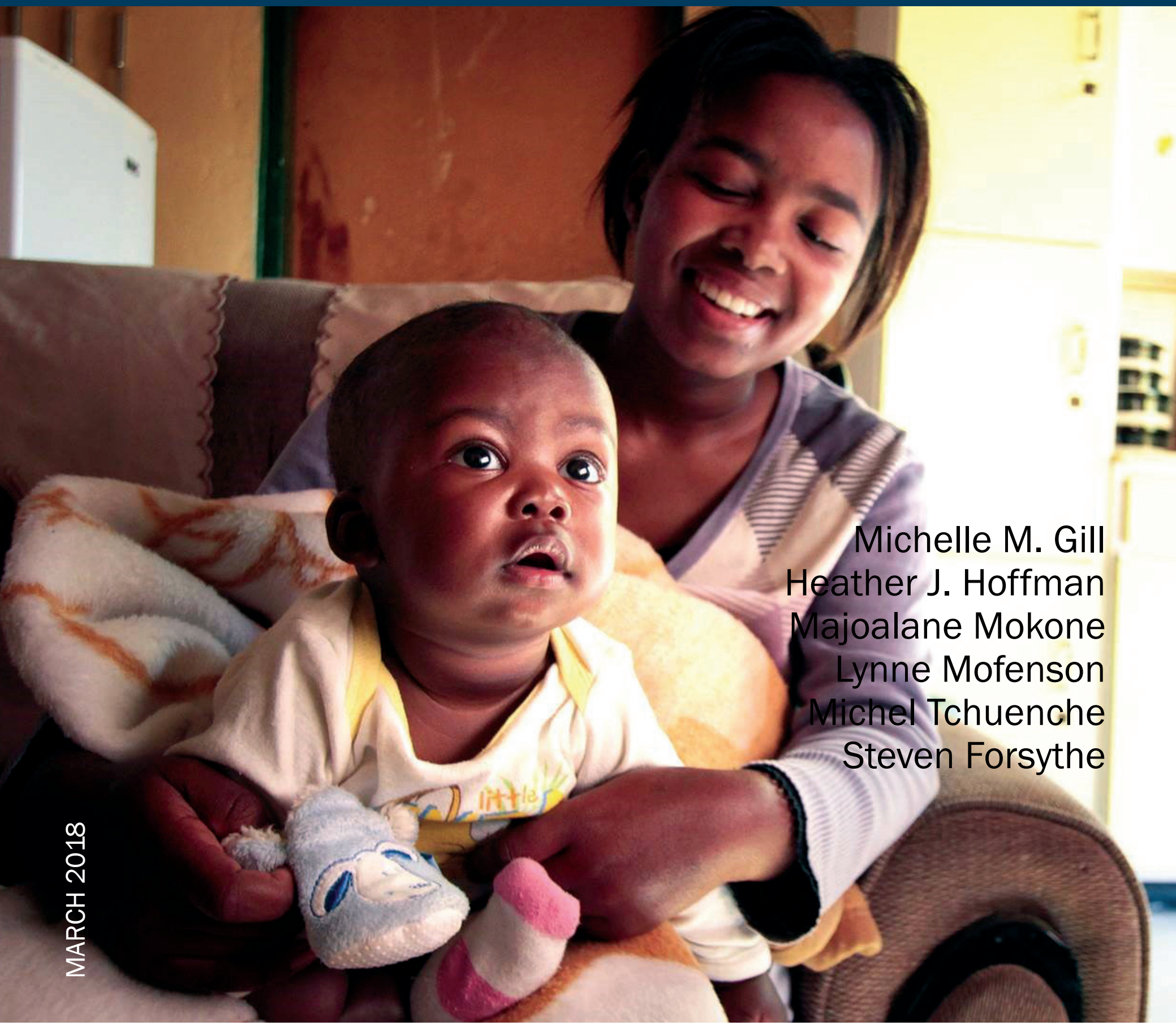




\section{Assessing the feasibility, acceptability, and costs of diagnosing HIV at birth in Lesotho and Rwanda}

Elizabeth Glaser Pediatric AIDS Foundation Michelle M. Gill Heather J. Hoffman Majoalane Mokone Lynne Mofenson

Avenir Health Michel Tchuenche Steven Forsythe 
Project SOAR

Population Council

4301 Connecticut Ave, NW, Suite 280

Washington, D.C. 20008 USA

Tel: +1202 2379400

Fax: +1 2022378410

projsoar.org

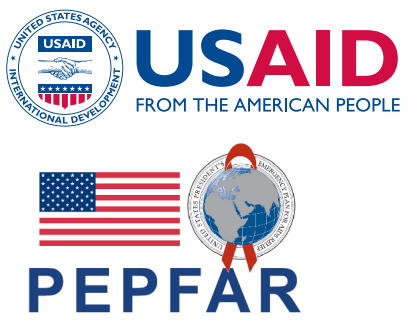

Project SOAR (Cooperative Agreement AID-OAA-A-14-00060) is made possible by the generous support of the American people through the President's Emergency Plan for AIDS Relief and the United States Agency for International Development (USAID). The contents of this guidance document are the sole responsibility of Project SOAR and the Population Council and do not necessarily reflect the views of USAID or the United States Government.

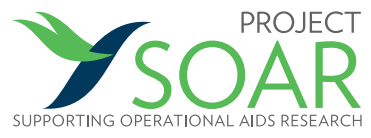

Through operations research, Project SOAR will determine how best to address challenges and gaps that remain in the delivery of HIV and AIDS care and support, treatment, and prevention services. Project SOAR will produce a large, multifaceted body of high-quality evidence to guide the planning and implementation of HIV and AIDS programs and policies. Led by the Population Council, Project SOAR is implemented in collaboration with Avenir Health, Elizabeth Glaser Pediatric AIDS Foundation, Johns Hopkins University, Palladium, and The University of North Carolina.

POPULATION COUNCIL

Ideas. Evidence. Impact.
Elizabeth Glaser Pediatric AIDS Foundation
Until no child has AIDS.
The Population Council confronts critical health and development issues-from stopping the spread of HIV to improving reproductive health and ensuring that young people lead full and productive lives. Through biomedical, social science and public health research in about 50 countries, the Council works with our partners to deliver solutions that lead to more effective policies, programs, and technologies to improve lives worldwide. Established in 1952 and headquartered in New York, the Council is a nongovernmental, nonprofit organization with an international board of trustees.

The Elizabeth Glaser Pediatric AIDS Foundation (EGPAF) is a nonprofit organization dedicated to preventing pediatric HIV infection and eliminating pediatric AIDS through research, advocacy, and prevention, care, and treatment programs. Founded in 1988, EGPAF supports activities in 19 countries around the world.
Avenir Health was founded in 2006 as a global health organization that works to enhance social and economic development by providing tools and technical assistance in policy, planning, resource allocation and evaluation. Avenir Health focuses on developing and implementing demographic, epidemiological and costing models for long-range planning to assist with setting goals, strategies, and objectives. Avenir Health assists in both developing and implementing programs in HIV/AIDS, reproductive health, maternal health and other programming areas, working with government agencies, foundations, corporations, and nongovernmental organizations around the world.

Published in March 2018.

(C)2018 The Population Council, Inc.

Suggested citation: Gill, Michelle, Heather Hoffman, Majoalane Mokone, Lynne Mofenson, Michel Tchuenche, Steven Forsythe. 2018. "Assessing the feasibility, acceptability, and costs of diagnosing HIV at birth in Lesotho and Rwanda," Project SOAR Final Report. Washington, D.C.: Population Council. 


\section{TABLE OF CONTENTS}

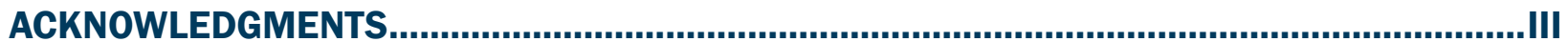

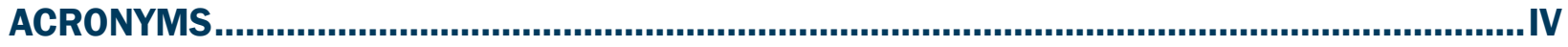

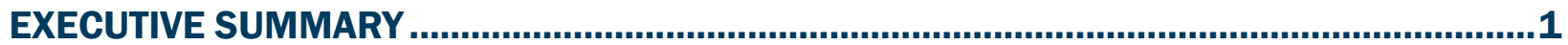

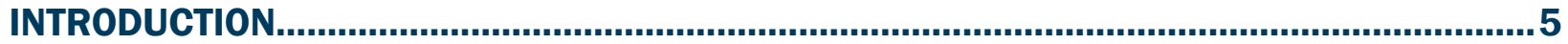

METHODOLOGY

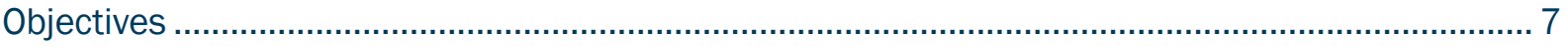

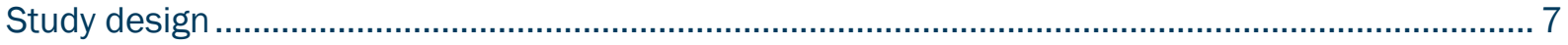

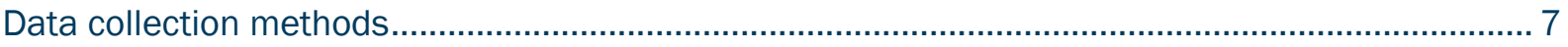

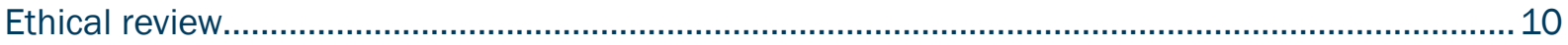

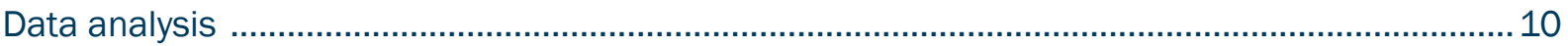

KEY FINDINGS

Characteristics of the semi-structured interview participants .......................................................12

Qualitative findings on acceptability and feasiblity ...................................................................12

Data abstraction findings: diagnosis and treatment turnaround times ........................................18

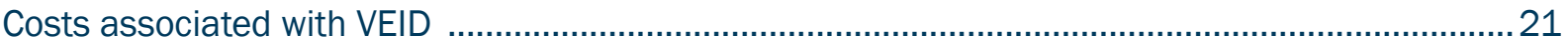

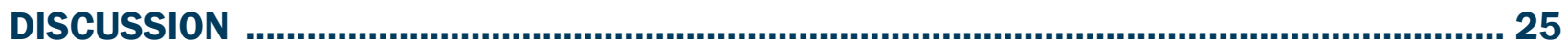

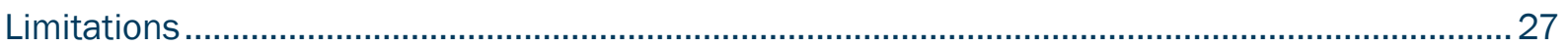

CONCLUSION AND RECOMMENDATIONS.......................................................................... 29

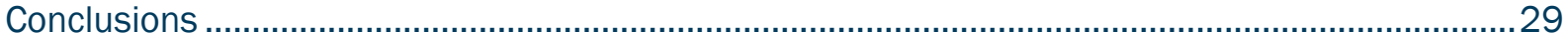

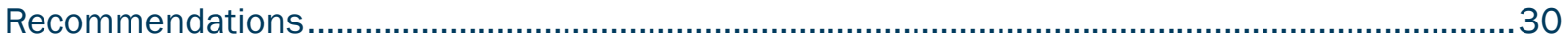

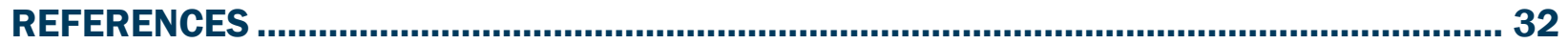

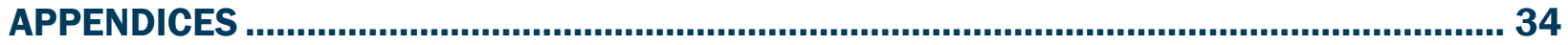

Appendix A

Illustrative quotes by thematic area of women, health care providers, and laboratory

personnel who participated in in-depth interviews.

Appendix B

Specimen transfer from facilities and TAT: responses from HWs and laboratory personnel 38 
Appendix C

TAT (in days) for PEA-WIL children infected with HIV since start of study tested via DNA-PCR ........40

Appendix D

Selected demographic and clinical characteristics of HIV-positive women and their infected infants in Lesotho $(n=11)$

Appendix E

Selected demographic and clinical characteristics of HIV-positive women and their infected

infants in Rwanda $(n=6)$

Appendix F

Costing: facility and laboratory surveys 


\section{ACKNOWLEDGMENTS}

This evaluation was conducted by the Elizabeth Glaser Pediatric AIDS Foundation (EGPAF) in collaboration with the George Washington University (GWU) with leadership on the costing component provided by Avenir Health. The authors would like to thank Vibhuti Haté for her technical support on the costing, Emma Sacks for assistance with the laboratory qualitative analysis, and the very early infant diagnosis (VEID) data collection team for all of their efforts: Liteboho Kali, Makara MotsoMoena, 'Mamosoeu Rose Khoete, Refiloehape Matsepe, Likano Phenya, and Limpho Sebotsa. We greatly appreciate the assistance provided by the Lesotho PEA-WIL Study investigators (complete list below) and the coordination and data team, including Sesomo Mohale, Mamello Masuoane, Matela Makhohlisa, Janet Barasa, as well as the PEA-WIL study Nurses who graciously added VEID data collection activities to their workload. We would also like to thank the Kabeho Study investigators in Rwanda, particularly Dieudonne Ndatimana and Gilles Ndayisaba, for filling in critical gaps in the data. We also wish to acknowledge the essential cooperation of the Lesotho Ministry of Health, Riders for Health, and DHL Lesotho. Special thanks to Roche Molecular Systems, Inc for the donation of the additional Roche Cobas Ampli-Prep/Cobas TaqMan HIV-1 test quantitative test v2.0 kits for birth DNAPCR testing. Finally, thank you to USAID and Population Council for their support throughout this study.

\section{VEID/PEA-WIL Study}

Michelle M. Gill, EGPAF US, VEID Co-PI and PEA-WIL Co-investigator

Lynne Mofenson, EGPAF US, VEID Co-PI

Appolinaire Tiam, EGPAF US, VEID Co-PI and PEA-WIL Co-PI

Lori Bollinger, Avenir Health, VEID Co-investigator

Laura A. Guay, GWU/EGPAF US, VEID Co-investigator and PEA-WIL Co-PI

Heather J. Hoffman, GWU, VEID Co-Investigator

Matsepeli Nchephe, Lesotho Ministry of Health ( $\mathrm{MOH})$

PEA-WIL Co-Investigator and VEID Co-Investigator

Mamakhetha Phalatse, Lesotho MOH, VEID

Co-Investigator

Seble Kassaye, Georgetown University, PEA-WIL Co-PI

Anthony Isavwa, EGPAF Lesotho, PEA-WIL Co-investigator

Mosilinyane Letsie, Lesotho MOH, PEA-WIL

Co-Investigator

Rhoderick Machekano, EGPAF US, PEA-WIL Co-Investigator

Florence M. Mohai, EGPAF Lesotho, PEA-WIL Co-

Investigator

Tsieso Motsoane, Lesotho MOH, PEA-WIL Co-Investigator

Vincent Tukei, EGPAF Lesotho, PEA-WIL Co-Investigator

\section{Kabeho Study}

Anita Asiimwe, Rwanda University Teaching Hospitals, Co-PI

Emily A. Bobrow, Co-PI

Placidie Mugwaneza, Rwanda MOH, Co-PI

Linda Adair, University of North Carolina, CoInvestigator

Cyprien Baribwira, University of Maryland, Baltimore Co-Investigator

Jeanine Condo, Ag. Principal of College of Medicine and Health Sciences, Co-Investigator

Michelle Gill, EGPAF US, Co-Investigator

Laura A. Guay, GWU/EGPAF US, Co-Investigator

Heather J. Hoffman, GWU, Co-Investigator

Emil Ivan Mwikarago, Rwanda Biomedical Centre, $\mathrm{MOH}$, Co-Investigator

Gilles F. Ndayisaba, EGPAF Rwanda, Co-Investigator

Sabin Nsanzimana, Rwanda MOH, Co-Investigator

Eric Remera, Rwanda MOH, Co-Investigator

Muhayimpundu Ribakare, Rwanda $\mathrm{MOH}$, Co-Investigator 


\section{ACRONYMS}

$\begin{array}{ll}\text { AIDS } & \text { Acquired immune deficiency syndrome } \\ \text { ANC } & \text { Antenatal care } \\ \text { ART } & \text { Antiretroviral treatment } \\ \text { DBS } & \text { Dried blood spot } \\ \text { DNA-PCR } & \text { Deoxyribonucleic acid-polymerase chain reaction } \\ \text { EGPAF } & \text { Elizabeth Glaser Pediatric AIDS Foundation } \\ \text { EID } & \text { Early infant diagnosis } \\ \text { GDP } & \text { Gross domestic product } \\ \text { HIV } & \text { Human immunodeficiency virus } \\ \text { HTC } & \text { HIV testing and counseling } \\ \text { HW } & \text { Health worker } \\ \text { ICER } & \text { Incremental cost-effectiveness ratio } \\ \text { IRB } & \text { Institutional review board } \\ \text { Kabeho Study } & \text { Kigali Antiretroviral and Breastfeeding Assessment for the Elimination of HIV } \\ \text { LIS } & \text { Laboratory information system } \\ \text { MCH } & \text { Maternal and child health } \\ \text { MOH } & \text { Ministry of Health } \\ \text { NRL } & \text { National Reference Laboratory } \\ \text { PEA-WIL Study } & \text { PMTCT Program Effectiveness among Women and Infants in Lesotho } \\ \text { PMTCT } & \text { Prevention of mother-to-child transmission } \\ \text { TAT } & \text { Turnaround time } \\ \text { USAID } & \text { United States Agency for International Development } \\ \text { VEID } & \text { Very early infant diagnosis } \\ \text { WHO } & \text { World Health Organization } \\ & \\ \text { HT } & \end{array}$




\section{EXECUTIVE SUMMARY}

HIV disease progresses much more rapidly in children than in adults. Mortality begins to rise in the first few weeks of life for HIV-positive infants not on ART. Infant HIV diagnosis as early as possible in a child's life followed by immediate ART could stem this progressive rise in infant mortality, particularly as several studies have suggested that an increased proportion of perinatal infections may occur in utero when maternal ART is received during pregnancy. This study, "Assessing the Feasibility, Acceptability, and Costs of Diagnosing HIV at Birth in Lesotho and Rwanda," leverages two existing cohort studies to address some critical questions related to very early infant diagnosis (VEID). The objective was to determine the feasibility, acceptability, and costs associated with the addition of birth HIV testing to the routine testing algorithm for infants born to HIV-positive women. Findings will contribute to the global guidance on whether current early infant diagnosis (EID) guidelines should be reconsidered to add birth HIV testing as standard of care.

\section{METHODOLOGY}

This study used a mixed methods design. Some existing data from two USAID-funded study cohorts in Lesotho and Rwanda were extracted for this analysis: the Prevention of Mother-to-child Transmission (PMTCT) Program Effectiveness among Women and Infants in Lesotho (PEA-WIL) Study taking place in three EGPAF-supported districts (Thaba-Tseka, Butha-Buthe, and Mohale's Hoek) and the Kigali Antiretroviral and Breastfeeding Assessment for the Elimination of HIV (Kabeho) Study. Birth DNA-PCR testing was added prospectively to the standard testing algorithm at ages 6 and 14 weeks in Lesotho. Retrospective DNA-PCR testing of dried blood spot specimens collected at or around the time of birth was performed in infants who participated in the Rwandan study.

New qualitative and quantitative study activities were also conducted under this VEID study. These include:

1. Semi-structured interviews with health workers (HWs), laboratory personnel, and a subset of postpartum women from the PEA-WIL study in Lesotho. Interviews were conducted with a total of 46 respondents from eight PEA-WIL facilities, five district laboratories, and the National Reference Laboratory (NRL). Data were coded according to a standardized codebook and analyzed according to the following themes: acceptability, feasibility, and retention and treatment initiation.

2. Abstraction of HIV and testing-related data from mother-baby pairs and dates of specimen and result transfer along the "EID pathway" from the parent study cohorts, PEA-WIL and nonPEA-WIL facilities, and the national EID database. Turnaround times (TAT) were calculated and compared at study and non-study facilities for HIV-exposed infants receiving birth and six-week DNA-PCR testing and for all HIV-positive study infants.

3. Interviews and record review of facility and laboratory costing data. Both direct (personnel, consumables) and indirect (personnel, capital costs, maintenance and utilities) costs were 
collected retrospectively from January to December 2015 at eight PEA-WIL facilities and the NRL. VEID unit cost, the additional cost of adding a test at birth, and the cost of identifying and initiating one child on treatment were calculated. Costing data collection took place in Lesotho only, though the unit cost from Lesotho was applied to the HIV transmission rate in Rwanda to assess the potential costs of offering VEID services in the Rwandan context.

\section{KEY FINDINGS}

\section{Qualitative findings on acceptability}

Overall, birth testing was acceptable to women, health workers (HWs), and laboratory staff participating in the in-depth interviews, with most recommending to roll out birth testing nationally. The major benefit cited by women and HWs was earlier knowledge of the child's HIV status. Additional benefits cited by HWs included earlier initiation of treatment for positive children resulting in a reduction in infant mortality, alleviation of caregiver uncertainty, and earlier test results allowing guidance on proper care for the child by mothers and HWs. All lab staff agreed that the primary benefit was early treatment initiation. Most women interviewed had no negative perceptions of VEID and generally expressed satisfaction with how services were delivered.

\section{Qualitative findings on feasibility}

HWs and laboratory staff reported that the DNA-PCR processing, transporting, and testing processes were the same at birth as other testing time points, and that some fast-tracking mechanisms were in place for positive test results, though these were not necessarily standardized or formalized. Feasibility-related challenges revealed in the interviews were largely reflective of the increased burden additional testing would have on existing shortcomings with the early diagnosis system as a whole, such as workload and reagent stock-outs, particularly at the central laboratory; problems with communication between facilities and laboratories; and delays in the return of HIV test results. Other challenges, such as missed opportunities to perform a birth test, could be attributed to the pilot nature of birth testing in the context of a study, which meant some activities (e.g., group counseling on VEID) were not routinized and that testing was only performed by study staff at facilities. Finally, the issue of home deliveries revealed weaknesses with tracing children to ensure they were tested within two weeks of life.

\section{Qualitative findings on infant retention and treatment initiation}

Women and HWs did not indicate that birth testing affected subsequent infant HIV testing acceptance or clinic attendance. HWs felt that clinic attendance would either not be impacted by the timing of infant diagnosis or that women who have infants infected at birth would have better attendance, given that they would be more highly motivated and need to return regularly for refills and more frequent monitoring. Women did not indicate that the addition of a birth test negatively influenced their motivations to attend standard clinic services. No respondents expressed a challenge with early initiation of treatment, but only the perspective of HWs was captured, as all women interviewed had HIV-negative children. HWs felt reactions did not vary among mothers 
learning their child was positive at birth versus other time points.

\section{VEID and EID turnaround times}

Findings on TAT from the data abstraction activity supported the interview responses regarding delays with specimen and results transfer. As expected, among infants at study facilities, those tested at birth received their results more quickly (starting from date of birth), with a median TAT of 74.5 days (IQR 47.5-100.0) compared to infants tested at six weeks, with a median of 103 days (IQR 92-116). At non-study facilities, the median TAT was 112 days (IQR 105-122) for the 6-week test. The maximum TAT to caregiver receipt of results was 125 days (approximately 16-18 weeks from the time of the birth test), so there were children likely having blood drawn again at a subsequent visit for their 6-week test without having yet received the results from the birth test. As indicated in the interviews, infants with positive HIV results were fast-tracked. The time from blood draw to ART initiation was 38 days for the 1 PEA-WIL study child identified as infected at birth, 49.0 days for the 1 PEA-WIL study child identified as infected at 6 weeks, and 35.0 and 66.0 for the 2 non-PEA-WIL children identified as infected at 6 weeks in the VEID study cohort. Overall, there were 11 HIV-positive infants in the PEA-WIL Study as of October 2016. The time from date of blood draw to ART initiation for the PEA-WIL study infants identified as infected at birth ranged from 35 to 62 days. Overall time from blood draw to ART initiation was 49 and 63 days for the 2 infants identified as infected at 6 weeks, 177 and 199 days for 2 out of the 3 infants identified as infected at 14 weeks, and 40 and 139 days for the 2 infants identified as infected at 6 months.

\section{Costing}

The comprehensive unit cost per VEID test (including counseling, drawing, packing and transporting samples, and performing the test at the NRL) in Lesotho was $\$ 40.50$. One of 199 infants was identified as HIV-positive at birth in Lesotho in 2015 (0.5 percent in utero infection rate) and 2 of 517 in Rwanda retrospectively in 2013 and 2014 (0.4 percent in utero infection rate). Based on the data from the VEID pilot study in Lesotho and the Kabeho study in Rwanda, the cost of identifying and initiating one infant on treatment was lower in Lesotho than in Rwanda: $\$ 8,057$ and $\$ 10,381$, respectively. The country cost per child benefiting from VEID was driven by the rate of in utero infection in children. For example, in Lesotho, costs ranged from $\$ 8,057$ with a 0.5 percent in utero infection rate to $\$ 810$ with a 5 percent in utero infection rate. Based on 2015 data, if the VEID program were rolled out nationally in Lesotho, in one year, there could have been as many as 37 HIV-positive infants who would have benefitted from VEID, and 8 deaths would potentially have been averted due to the VEID services and early ART initiation. In Rwanda, in one year, 22 infants would have benefitted from very early diagnosis and ART initiation, and 5 deaths would potentially have been averted.

\section{CONCLUSION AND RECOMMENDATIONS}

Should birth testing be implemented nationwide in Lesotho, findings from this study suggest that women, health workers, and laboratory personnel would find this policy change acceptable. They also indicated that subsequent infant HIV testing and postpartum clinic attendance would not 
be affected by adding birth testing to the diagnostic algorithm. However, this study illuminated some challenges to the feasibility of this approach, particularly with regards to the time from HIV testing to the return of test results and treatment initiation for HIV-positive infants. While infants identified as infected at birth were initiated on treatment more quickly than those tested at six weeks, the turnaround time was still long and varied between one and two months. In order to reduce TAT, it may be less burdensome and costly to strengthen counseling, tracing, and testing efforts around infant diagnosis beginning at six weeks of life rather than adding birth testing. Decentralization of testing to the district level and use of point-of-care HIV diagnostic machines might also permit more timely treatment initiation for infants identified as HIV-positive and thus minimize HIV-associated morbidity and mortality. Decentralized testing, particularly with point-ofcare machines that non-laboratory clinic staff can be trained to operate, could also reduce the burden to the health system when testing is conducted at a single national laboratory, though the costs of this approach also need to be considered.

In terms of VEID cost, costs are lowest when the rate of in utero infection is high. As VEID coverage increases, the number of infants benefiting from the service increases as well as the number of early infant deaths averted. In situations where PMTCT programs are not well-established and maternal ART use is low, and hence the risk of MTCT remains relatively high, universal birth testing might be considered. In situations where PMTCT programs are well-established and most women are on ART during pregnancy, and hence risk of MTCT is low, a targeted testing program of infants at high risk of in utero infection (e.g., no maternal antiretroviral therapy or less than four weeks of treatment during pregnancy) might be a costeffective way of implementing birth testing. However, a targeted program may be more complex to implement on a national scale, which could result in high-risk infants not being identified and hence missing birth testing; countries will need to consider both costs as well as implementation feasibility in designing national programs. 


\section{INTRODUCTION}

Global efforts to prevent mother-to-child HIV transmission (PMTCT) have resulted in a 70 percent decrease in new pediatric infections since 2000. Despite the scale up of PMTCT programs, almost 25 percent of HIV-positive pregnant women did not receive antiretroviral therapy (ART) in 2015, and treatment drop-out rates among women who were pregnant and breastfeeding remained high, leading to increased risk of transmission to their children (UNAIDS 2016). Moreover, there is still a widespread need for family planning in many countries (UNAIDS 2016, Campbell et al. 2016). Thus, it can be anticipated that new pediatric HIV infections will continue to occur for many years.

HIV disease progresses much more rapidly in children than in adults. Without ART, mortality is as high as 68 percent by age two in infants with perinatal infection, with much of this risk occurring within the first months of life (Newell et al. 2004, Marinda et al. 2007). Mortality begins to rise in the first few weeks of life, with an early peak as high as 20-30 percent between 8 and 12 weeks of age (Marinda et al. 2007, Marston et al. 2011, Bourne 2009). This rapid increase in mortality is due to a progressive decline in CD4 cell count and rapid HIV disease progression observed in young infants without ART (Violari et al. 2008, Innes et al. 2014).

Early infant HIV diagnosis (EID) is critical, as early ART initiation between ages 6 and 12 weeks has been shown to dramatically reduce early infant mortality by 76 percent and decrease HIV progression by 75 percent (Violari et al. 2008). However, a study of South African infants starting ART at a median age of 8.4 weeks showed that 62 percent had advanced HIV disease (CD4 <25 percent or WHO Stage 3 or 4) before starting ART even at this very early age (Innes et al. 2014). Thus, even earlier diagnosis, potentially in the first 48 hours of life, and immediate ART might be desirable. Several studies suggest that an increased proportion of perinatal infections may occur in utero when mothers are receiving antepartum ART, and hence can be detectable on day one of life (Lillian et al. 2013, Magder et al. 2005).

The current World Health Organization (WHO)-recommended algorithm for EID includes initial viral testing of HIV-exposed infants at age 4-6 weeks. However, in 2015, among the 21 Global Plan countries in Africa, only half of exposed infants received an HIV test in the first two months of life. Even in countries with higher EID coverage such as South Africa, due to delays between testing and return of results (turnaround time [TAT]), as many as 50 percent of infants tested were either not started on ART or were lost to follow-up (Smith et al. 2014). In many cases, by the time results are returned, the infant has already died. Very early infant diagnosis (VEID) and immediate ART have the potential to prevent decline in immunologic function and clinical deterioration, averting the progressive rise in infant mortality within the first weeks to months of life. A recent analysis from South Africa concluded that six weeks may no longer be the optimal age to diagnose perinatal HIV infections, and recommended consideration of two early PCR tests (VEID at birth, followed by a test at 10 weeks) as the ideal diagnostic algorithm (Lillian et al. 2014). 
While birth virologic testing appears to be an attractive intervention to reduce early HIV-related mortality, there remain many questions about implementing this approach on a broad scale, including the cost-effectiveness of adding another virologic test to the diagnostic algorithm.

The current study, Assessing the Feasibility, Acceptability, and Costs of Diagnosing HIV at Birth in Lesotho and Rwanda, leverages two existing USAID- funded EGPAF studies to address some of the questions related to VEID. In Lesotho, as part of a pilot evaluation, birth DNA-PCR testing was added prospectively to the standard testing algorithm at age 6 and 14 weeks at 13 study facilities in three districts. These facilities were participating in an observational prospective cohort study evaluating the effectiveness of PMTCT service delivery, the PMTCT Effectiveness among Women and Infants in Lesotho (PEA-WIL) study. In Rwanda, retrospective DNA-PCR testing of dried blood spot (DBS) specimens collected at or around the time of birth was performed in infants who participated in a study evaluating 24-month HIV-free survival in infants born to mothers receiving ART, the Kigali Antiretroviral and Breastfeeding Assessment for the Elimination of HIV (Kabeho) study. The goal of the current study was to utilize data from these two studies to evaluate the feasibility, acceptability, test TAT, and costs associated with the addition of birth HIV testing to the routine testing algorithm for infants born to HIV-positive women. The costing component was led by the Avenir Health team with the support of the EGPAF teams in Lesotho and Rwanda.

With approximately 30 percent of women aged 15-49 years estimated to be HIV-positive in Lesotho and nearly 4 percent in Rwanda, this study reflects both high and low HIV prevalence settings (Lesotho MOH 2016, Rwanda MOH 2015). PMTCT services are integrated in almost all antenatal facilities in both Lesotho and Rwanda. Both parent studies included a cohort of over 600 HIV-positive women. Children in the Kabeho study were born between April 2013 and April 2014 and the study was completed in August 2016. The last PEA-WIL delivery of an HIV-exposed child took place in October 2016 and prospective follow up of the cohort is ongoing. 


\section{METHODOLOGY}

\section{OBJECTIVES}

The objectives of the VEID study were to determine the:

1. Feasibility of adding early infant diagnosis testing at birth to the HIV diagnostic algorithm for infants born to HIV-positive women in selected facilities in Lesotho.

2. Acceptability of infant birth testing among health workers (HWs), laboratory personnel, and HIV-positive women in selected facilities in Lesotho.

3. Age at initiation of ART in HIV-positive infants with and without the availability of birth testing and the time from HIV diagnosis to ART initiation in selected facilities in Lesotho.

4. Unit cost per VEID test, the cost per early infection identified and initiated on treatment in Lesotho and Rwanda, and the potential impact of VEID in terms of early infant deaths averted.

\section{STUDY DESIGN}

This study used a mixed methods design. Some existing data from the USAID-funded study cohorts in Lesotho and Rwanda were extracted for this analysis. New qualitative and quantitative study activities were also conducted under the VEID study. These three activities were: 1) Semistructured interviews with HWs, laboratory personnel, and a subset of PEA-WIL study women (Lesotho only); 2) Abstraction of HIV and testing-related data from mother-infant pairs and dates of specimen and result transfer along the "EID pathway" from the parent study cohorts, PEA-WIL and non- PEA-WIL facilities, and the national EID database in Lesotho; and 3) Interviews and record review of facility and laboratory costing data. Costing data collection took place in Lesotho only, though the costing analysis included both countries. The VEID study did not introduce any new interventions, because birth DNA-PCR testing (defined as testing at birth or within the first two weeks of life) was already being conducted as part of the parent studies.

\section{DATA COLLECTION METHODS}

\section{Data Collection Activity \#1: Semi-structured interviews with HWs, laboratory personnel, and a subset of PEA-WIL study women in Lesotho}

Interviews were conducted to address objectives 1 and 2 on feasibility and acceptability of VEID, respectively. Interviews took place in eight out of the 13 PEA-WIL study facilities. Study facilities were located in Thaba-Tseka, Butha-Buthe, and Mohale's Hoek districts in Lesotho, representing the highlands, lowlands, and foothills regions, respectively. The subset of facilities were purposively selected for the VEID Study to reflect level (hospital, health center), geographical region (highlands, foothills, and lowlands), and public or private ownership (Table 1). Table 1 also shows which data collection activities took place at which facilities. 
Table 1 PEA-WIL/VEID study facilities in Lesotho: Characteristics and data collection activities

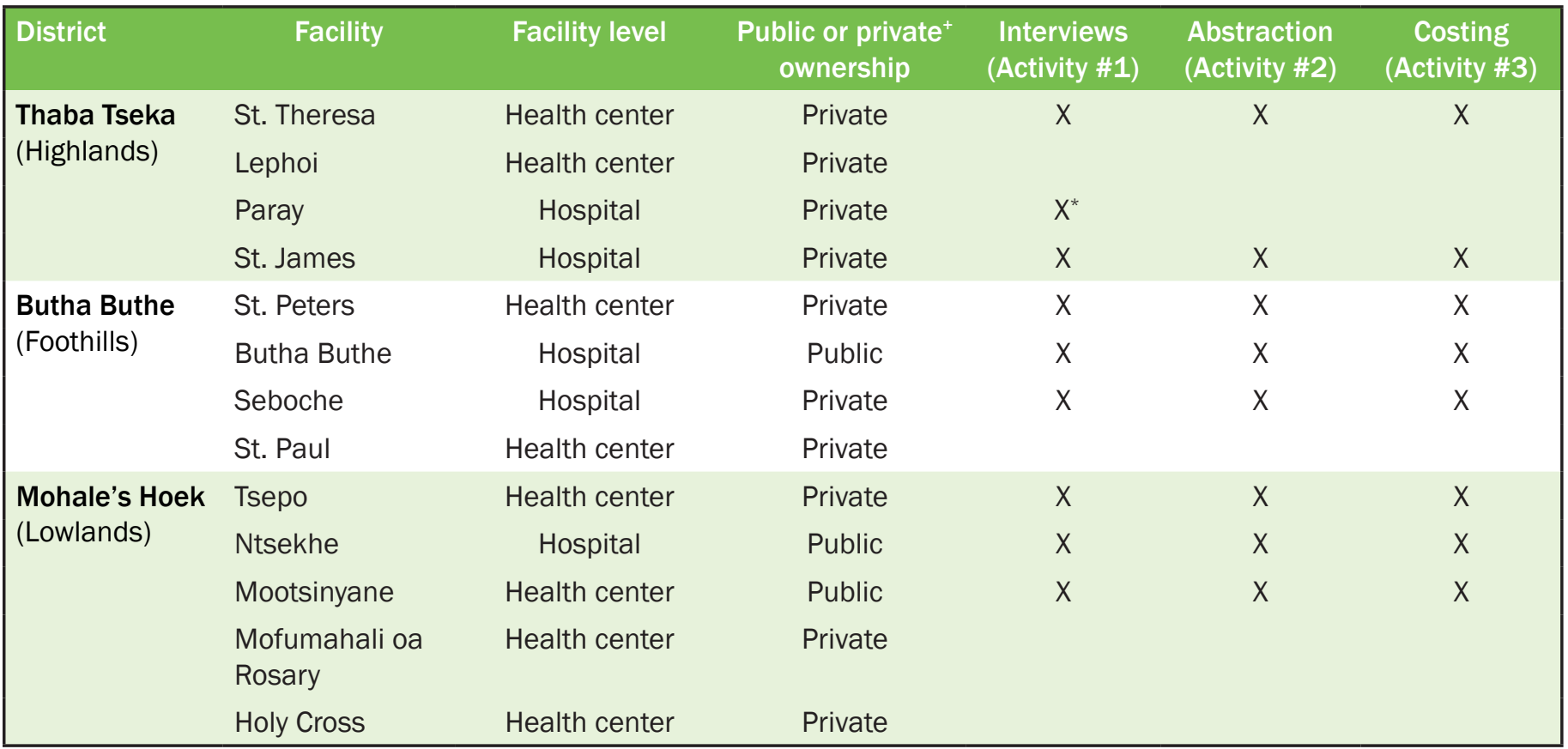

${ }^{+}$All private facilities were operated by Christian Health Association of Lesotho (CHAL).

*Lab personnel only

Women were eligible for the study if they were enrolled as PEA-WIL study participants and attending their 6-week or 14-week postpartum visit. However, any woman in the study with a child who was diagnosed as HIV-positive at birth regardless of visit timing was also recruited. One to four women's interviews were conducted per facility. For women's interviews, PEA-WIL study nurses communicated with research assistants when eligible women were scheduled for their visits so that they could travel to the respective districts to recruit potential study participants.

HWs were eligible for the study if they were working at a PEA-WIL study facility and involved in some aspect of VEID. HWs were excluded if they did not have involvement in any aspect of VEID. As birth testing was being piloted through the PEA-WIL study, only study nurses who performed all VEID-related tasks, and lay counselors who often delivered test results to the mother/caregiver, met this criteria. Clinic nurses were not eligible, as they were not involved in any aspect of VEID during this pilot phase. One to four provider interviews were conducted per facility. Laboratory staff were recruited for interviews if they were working in one of the study districts or study hospitals or the Lesotho National Reference Laboratory (NRL), and responsible for performing the tests. One staff member at each of the district laboratories and four NRL staff were enrolled. Potential HWs and laboratory participants were sequentially recruited based on their availability and days on which research assistants were present.

All interviews were conducted between March and July 2016 by research assistants trained in human subjects protections, the study protocol, and qualitative methods. The duration of the interviews was 30 minutes to 1 hour. Those involving PEA-WIL cohort women were conducted in Sesotho while interviews with HWs and laboratory staff were conducted in English. Topics 
included early testing and treatment acceptability, procedures (e.g., testing, preparing specimens, returning results, ensuring treatment initiation), and processes for home deliveries and false positive test results.

\section{Data Collection Activity \#2: Abstraction of HIV and testing-related data from mother-infant pairs}

Data were abstracted primarily in order to determine the timing of ART initiation for HIV-positive infants with and without the availability of birth testing in Lesotho (objective 3 ). This activity also served to compare whether or not TAT of receipt of results by the caregiver and/or ART initiation differed between study and non-study facilities, as the study facilities may have benefited from additional study-related support and attention compared to non-study facilities. After excluding low volume and hard-to-reach facilities (as was done for PEA-WIL site selection) in the same three study districts, the remaining ten EGPAF-supported non-study facilities were selected for inclusion in the VEID study. Finally, the demographic and clinical profiles of all PEA-WIL and Kabeho study women with HIV-positive children (at birth and other time points) were reviewed for any trends that might reveal risk factors for those whose children were infected at birth. The two subactivities are described below:

a. Calculate TAT for ART initiation for HIV-positive infants and other interim steps along the VEID pathway for HIV-exposed infants

All HIV-exposed infants in the subset of eight PEA-WIL facilities born between 12 January (following MOH IRB approval of the VEID sub-study) and 16 June 2016, to allow for approximately six months of data collection, were included for this activity. The one study child infected at birth during this timeframe was tested at a study facility not included in the subset; however, in order to have a comparison of TAT at birth versus six weeks, this child's information was included in the analysis. All children born to HIV-positive mothers who received a 6 -week DNA-PCR test at 10 selected non-PEA-WIL study facilities in the 3 study districts during the same time period were also included. PEA-WIL children contributed data for both their birth and six-week DNA-PCR tests. In order to construct TAT, dates for the time of blood draw, specimen receipt and testing at NRL, result receipt at health facility, receipt of result by caregiver, and ART initiation (if applicable) were either extracted from the national EID database or PEA-WIL study database or abstracted from facility registers. Data were collected by trained research assistants and members of the study coordination team using paperbased tools between July and September 2016. All completed data were reviewed by the study coordinator before entry into an Access database.

b. Review demographic and clinical profiles of all women with HIV-positive children from the PEAWIL and Kabeho cohorts and calculate TAT for all HIV-positive children diagnosed by DNA-PCR testing in Lesotho

All PEA-WIL and Kabeho mother-infant pairs were included if the child was diagnosed as HIVpositive at any testing time point. This includes all Kabeho pairs and any diagnoses among PEA-WIL pairs up until October 2016. This activity was undertaken to help address the question of whether or not selective birth testing would be appropriate based on maternal factors such as HIV diagnosis timing, ART duration, adherence, and viral load results. These and other data were extracted from the parent study databases. As not all PEA-WIL HIVpositive infants were captured in the activity described in (a) above, this exercise ensured that 
TAT was included for all infants diagnosed with DNA-PCR testing. The national EID database and facility records were also utilized for this activity.

\section{Data Collection Activity \#3: Interviews and record review of facility and laboratory costing data}

This activity addressed objective 4 on determination of costs associated with the addition of a birth test to the EID algorithm. Eight sites were purposively selected as described above. Data collection tools were piloted at one health center in Mohale's Hoek and at a district laboratory in Thaba Tseka; neither of these facilities was included in the subset. Responses from the pilot testing were documented and the data collection tools were subsequently revised and resubmitted by EGPAF for ethics approval.

Cost data from these eight facilities and from the NRL were collected retrospectively for the January-December 2015 time period. Staff interviews were conducted with finance/account officers, facility managers, and nurses involved in VEID at facilities and the laboratory staff processing and performing the DNA-PCR test. Both direct (direct personnel, consumables) and indirect (indirect personnel, capital costs, maintenance and utilities) costs were collected at the eight study facilities as well as at the NRL. These figures do not include the costs of pediatric treatment, PMTCT, training, external technical assistance, or VEID-associated research activities. As stated above, no staff were specifically trained to perform VEID during the period covered in this study (January to December 2015), given that staff were already trained to perform virologic EID testing of infants. In most cases, interviewees were not willing to share any salary information. Robust effort was made by the study team to obtain salary grades, which were used in lieu of the exact salary.

\section{ETHICAL REVIEW}

This study was approved by the Ministry of Health Research and Ethics Committee and the George Washington University Institutional Review Board (IRB) in Washington, DC. All study staff involved in data collection received human subjects' research ethics and training on the informed consent process prior to being in contact with the participants. This study was submitted as an amendment to the PEA-WIL Study.

\section{DATA ANALYSIS}

\section{Data Collection Activity \#1}

Four research assistants (interviewers) simultaneously transcribed and translated any Sesotho recordings into English in MS Word files in preparation for qualitative analysis. Subsets of recordings were reviewed against the transcripts by study staff fluent in both English and Sesotho. Transcripts were reviewed by the Co-Principal Investigator (Gill) as they were finalized. Translated transcripts were imported into MAXqda (V10), a qualitative data analysis program. Data were coded according to a standardized codebook by the same four research assistants. A small 
subset of transcripts were coded by multiple reviewers and questionable segments of coded text were resolved among the coders or by the Co-PI. Data were analyzed according to the following themes: acceptability, feasibility, and retention and treatment initiation. Data were reduced to matrices, then textual summaries, with quotes identified to illustrate key or novel points.

\section{Data Collection Activity \#2}

Total TAT from sample collection to caregiver result receipt or ART initiation for HIV-exposed and positive infants, respectively, were calculated along with TAT for intermediate steps along the VEID pathway. Descriptive statistics were calculated for all variables of interest using frequencies and percentages for categorical variables and means (standard deviations) and medians (interquartile ranges) for continuous variables. The data analysis was conducted using SAS/STAT software, Version 9.4 of the SAS System for Windows (SAS Institute Inc., Cary, NC, USA).

\section{Data Collection Activity \#3}

Two Excel spreadsheets were customized to calculate key outputs: the unit cost of providing VEID in Lesotho, the cost associated with the addition of an HIV test at birth, and the cost per early infection identified and treated. The 2015 average exchange rate applied was LSL12.77 per US dollar ${ }^{1}$. Input costs shared with other services were allocated to the VEID program based on the proportion of the number of VEID patients to the total number of patients seen at the facilities.

A costing exercise was not performed in Rwanda, but the unit cost from Lesotho was applied to findings from the Kabeho study to assess the potential financial costs of offering VEID services in Rwanda, a lower HIV prevalence country.

${ }^{1}$ http://www.oanda.com/currency/average (2015) 


\section{KEY FINDINGS}

\section{CHARACTERISTICS OF THE SEMI-STRUCTURED INTERVIEW PARTICIPANTS}

Semi-structured interviews were conducted with 20 HIV-positive women who were attending their 6-week ( $n=13$ ) or 14-week $(n=7)$ postnatal visit. Of the 13 women interviewed at 6 weeks, 5 had not yet received infant birth testing results. Of the 7 women interviewed at 14 weeks, 1 did not receive a birth test for her child because the testing window was missed following a home delivery; the other 6 women received their infants' birth results. Seven infants had blood taken on the day of birth, with the remaining tested a few days post-delivery or at the 7-day visit. All infants had blood taken for a 6-week test and 4 women attending the 14-week visit received these results. All test results were HIV-negative.

Seventeen HWs responsible for administering birth testing or delivering birth test results were interviewed. Fourteen HWs were study nurses (two of whom were trained as midwives) and four were counselors. Years of professional experience ranged from less than 1 year to 15 years, with a median of 3 years (IQR 2-9). The start date for birth testing implementation varied in their facilities, from June 2014 to February 2015. Five district laboratory staff and four central laboratory staff were interviewed; five were lab technicians and four were lab technologists.

\section{QUALITATIVE FINDINGS ON ACCEPTABILITY AND FEASIBLITY}

This section presents key findings organized by the following themes: acceptability, service retention and uptake, facility feasibility, and laboratory feasibility. Illustrative quotes can be found in Appendix A.

\section{Acceptability}

Acceptability has been defined by this study using the constructs of perceived benefits and challenges of early testing and treatment initiation, birth test refusals, and perspectives on national policy recommendations, including selective ("high risk") versus universal testing.

\section{VEID benefits}

Nearly all women interviewed stated they were happy to have their child tested or to know their child's HIV status at birth. The primary benefit cited by women and HWs was earlier knowledge of child HIV status. HWs added that birth testing allowed them to initiate earlier treatment, reduce infant mortality, alleviate caregiver uncertainty, and guide proper care. All lab staff agreed that the primary benefit was early treatment initiation. Most women interviewed had no negative perceptions of VEID. 


\section{VEID challenges}

While women thought it was important to have their children tested at birth, they still expressed concerns about testing that were not specific to timing, but in regards to possible infant transmission, except for two women who expressed discomfort over pricking their newborn to obtain blood. Women, HWs, and lab staff cited TAT as a challenge for all DNA-PCR tests-one woman in particular pointed out that her child was being tested again at six weeks, without yet receiving the birth test results.

Nearly all lab respondents were concerned about the delays to TAT that would potentially result if birth testing were implemented nationwide. One delay was related to the transportation system for specimens and results. Since some clinics have set days for blood draws, and transportation for routine services at facilities is typically Monday through Friday, lab staff worried that for those women who deliver on a weekend or after hours, the sample could remain at the facility for 5-6 days before being picked up. One district respondent suggested the pick-up schedule should be twice per week, which is already happening in some facilities. Another TAT-related concern for the lab was the limited capacity of the machines, which can only run so many tests at one time. Additional TAT challenges are listed in the next section.

Other challenges reported by HWs and lab staff included difficulty obtaining a sufficient blood sample at birth and weak follow-up systems to capture women who deliver at home, whose infants then may miss the opportunity to receive a birth test. HWs also described issues that were reflective of the pilot nature of birth testing, which could be addressed if the algorithm was updated in the national guidelines. These included lack of birth testing information in standard facility registers and group counseling messages that omitted birth testing and failed to emphasize the 7-day visit for infants not tested at birth. Other lab-specific concerns included staff shortages, stock-outs (mostly at central level), and storage of additional samples at the NRL.

While HWs did not perceive a significant increase in resource burden as part of the pilot, counselors and all lab staff felt if birth testing were rolled out there would be an increase in workload, which could be addressed at the laboratory level by the addition of lab technologists, or data clerks to assist with the registration and packaging of samples.

\section{Birth test refusals}

HWs reported that birth testing refusals were rare; those that encountered refusals said the reasons were 1) fear of hurting newborn, 2) long waiting time for blood draw, and 3) fear of HIVpositive result. VEID acceptance was sometimes the result of extensive counseling as well as the existing, trusting relationship between study nurse and patient.

\section{National policy recommendations}

$\mathrm{HWs}$ felt that birth testing was important and the majority recommended nationwide implementation. Most HWs and lab staff recommended birth testing for all HIV-exposed children, rather than selective "high risk" testing, reasoning that all HIV-positive women should be considered high risk and it is not always possible to know who is adherent to treatment. While one lab respondent was in favor of broader implementation, it was suggested that VEID rollout be 
staggered, starting with hospitals and then lower level facilities, in order to better understand the extent of the added burden to the lab/health system and to plan accordingly.

\section{Feasibility}

Feasibility has been defined by this study as the procedures used for HIV birth testing during the parent study and how well they were performed, including counseling on and understanding of birth testing, specimen transfer and processing, relaying results (including TAT and fast-tracking HIV-positive results), and addressing the issues surrounding home deliveries.

\section{Counseling on and understanding of birth testing}

Women seemed to have some difficulty in understanding what was meant by counseling during the interviews. The majority of respondents described counseling in basic terms, that they were told blood was drawn to determine if their child was infected with HIV, but few other details were mentioned. According to the counseling experiences described by women, two had nurses try to prepare them for whatever result was received, one woman received an explanation about how the blood was to be drawn, another was counseled on the series of tests the infant would undergo (though she could not recall this accurately), and finally one was told her child was being tested for the purpose of the study. From the HWs perspective, approximately half said their counseling included the importance of testing the child as soon as possible after birth, in order to initiate treatment and in effect save the child if positive. Other HWs reported more general strategies including the modes of transmission, testing algorithm, when to expect results, the logistics of testing, and, in a few cases, what would happen if the child tests positive for HIV. Nurses and counselors also talked about the importance of ensuring the woman felt it was her choice and that permission was granted, but that women may need significant time to consider the test and discuss it with their health care provider, particularly if they just gave birth.

According to HWs, the most frequently mentioned questions and concerns by women were what to do if their child's result was positive, when results could be expected, would the child be hurt unnecessarily, and that the child was too young to have blood drawn. Only one woman said that not all of her questions were answered. HWs generally felt as if their patients understood the counseling on birth testing clearly. They pointed to the discussions held, questions asked, and that women would even be the ones to remind nurses about testing at various time points as evidence of their understanding.

A significant number of women described the time spent counseling as too short. Among these women, the amount of time being counseled varied from 5 to 35 minutes. No counseling was reported to take longer than an hour. If just focusing on the time it took to counsel on birth testing immediately before the test, three HWs responded with a range of 5-20 minutes. When estimating across ANC and delivery visits, HWs estimated anywhere between 15 to 60 minutes, depending on the woman's level of understanding and questions, with the majority of HWs estimating closer to 15-30 minutes.

Almost all HWs indicated that women were prepared for birth testing since its purpose and importance was conveyed in group education and explained during the consent process at study 
enrollment, and women received reminders about the infant testing schedule during follow-up ANC visits. Most women also reported that they were made aware of birth testing during their antenatal visits, and all women who were asked said that the information they received about birth testing in ANC versus delivery was consistent. When women were asked about counseling for subsequent DNA-PCR testing (at 6 or 14 weeks), approximately half of respondents reported that they did not receive counseling before blood was drawn.

\section{Specimen transfer and processing and relaying results}

HWs and lab staff described the steps from blood draw through receipt of results back at the facility. In general, infant blood collection involves pricking children on the heel or toe with a lancet and then bringing the DBS filter paper to the foot so that blood can be filled neatly within the five circles of the card. Collection of birth specimens was performed in the delivery ward or postnatal room by PEA-WIL study nurses, who were also generally responsible for specimen preparation and packaging and documentation. If the district lab and $\mathrm{MCH}$ clinic were located on the same hospital campus, nurses would hand-deliver specimens to the district lab; for most health centers, Riders for Health transported the specimens to the district labs using motorbikes. District laboratories registered specimens and compiled with others before shipping to the NRL via DHL (usually weekly), or sometimes by hospital vehicle. District laboratories did not perform DNA-PCR testing. One respondent reported that the registration step was sometimes skipped. All samples were supposed to be registered in the Laboratory Information System (LIS), which should be linked to the NRL, but this feature was not functional at the time of interviewing.

At the NRL, patient/specimen information and results were logged into the LIS. Prior to processing, the DBS samples were prepared manually. A series of quality control steps were performed to ensure accuracy. The machine logged the time and date and created hard copy printouts. A second person had to review the results before authorizing them. Printouts of results were typically returned via DHL to district laboratories and then in turn by Riders to health centers. Study nurses or HIV testing and counseling (HTC) counselors delivered results to caregivers.

The frequency of specimen and result transport and the average TAT for some of these activities are provided in Appendix B. There were some differences in responses among HWs and lab staff at the same facility in terms of how often specimens were transferred to the district lab and how often they were picked up to be taken to the NRL. Estimated TAT to receive results back at the $\mathrm{MCH}$ clinic ranged from two weeks to three months at both health centers and hospitals. Some respondents indicated that sometimes results were never received. The variability and uncertainty made it difficult to know when to tell women when to return to pick their results; thus HWs instructions varied as well (e.g., check every time at clinic, in one month, at next visit). Given the expense and delay of transporting samples and results, multiple HWs and lab respondents suggested machines to run the tests be placed in districts in order to bring EID services closer to women. Other suggestions to reduce TAT for all DNA-PCR testing included:

- Communicate HIV-negative results to women via text messaging and include prevention strategies for keeping their child negative. 
- Facilitate better communication between the NRL and MCH clinics by providing a phone at the central lab so that documentation errors can be addressed more quickly, as emails are not always checked and access to the internet is limited in some places.

- Call the MCH clinic immediately whenever there is a positive result or repair the SMS printers so there is a paper trail for results.

- Ensure the LIS is working properly between the district and central laboratory levels so that when results are returned, the district lab can simply send them along to clinics.

Nearly all facilities described how HWs attempt to fast-track HIV-positive results to the caregiver. Once a positive result reaches the facility, the first step is typically for the nurse or counselor to call the caregiver. As one nurse in a hospital put it, "all people in $\mathrm{MCH}$ who saw the results that are positive, it's their responsibility to call that lady." If the caregiver cannot be reached by phone, a community or village heath worker will conduct a home visit or look for the woman in the surrounding villages and ask her to return to the health facility. A respondent at one hospital indicated that $\mathrm{s} /$ he tried to get caregivers to the clinic within a day. Fast-tracking was contrasted with what happens when there is a negative result, which is to wait until the woman attends her regularly scheduled clinic visit, since they are coming in every 1-2 months anyway. Women described this approach in their interviews as working well.

The HW-reported challenges with following up with women whose infants had an HIV-positive test result included poor mobile network coverage, unavailable numbers, and reaching caregivers who were not local. While there may be fast-tracking mechanisms on the part of facility staff, there does not appear to be a formal fast-tracking mechanism once it is discovered that the result is positive at district or central laboratory level, though lab staff from three hospitals reported having walked over the paper result to the $\mathrm{MCH}$ clinic on its campus or calling the health center if there is a positive result, rather than waiting for nurses or Riders to pick up results. However, most communication is in the direction of the health facility to the lab (via the toll-free number) when results have been delayed. The reason given for results not typically being relayed over the phone is so that the results can be properly documented in the patient's health booklet. One respondent described an instance where a positive result was communicated via phone between district lab and health facility, but the result was actually negative and the child was initiated on ART unnecessarily.

\section{Facility-level birth testing differences}

HWs and lab respondents reported that there were not differences in the DNA-PCR testing process that takes place at birth versus other time points. There were also no differences found between birth and subsequent DNA-PCR testing with regards to TAT. The only distinction made was a function of the study itself. PEA-WIL nurses were often the only cadre at the health facility to draw birth specimens; at other time points, this may be done by HTC counselors. Given this, several respondents recommended that all nurses and counselors be responsible for birth testing and are afforded the proper training to do so.

\section{Home delivery follow-up}

As stated above, home deliveries were named as a birth testing challenge. Women may not return to the facility within the two-week window to have their infant tested and systems needed 
strengthening in order to follow up with women who deliver at home, in South Africa, or, in the case of this pilot, deliver at a non-study facility, which did not offer birth testing. If rolled out nationally, it was also felt that health education would need to be improved to help ensure mothers deliver in health facility and birth DNA-PCR tests are not missed.

Nurses employed several different strategies to ensure a birth test was conducted, the most common of which was use of village or community health workers to bring women and their newborns to a health facility. Lay staff may accompany women who deliver at home to a health facility, report such instances to the $\mathrm{MCH}$ clinic, or track these mother-baby pairs and bring them in once a nurse has learned of the delivery. Other strategies included emphasizing the importance of facility delivery, or in cases where home delivery could not be avoided, bringing the child to the health facility as soon as possible, requesting that the nurse be notified in the case of home delivery, and following up with women via phone around the time of their estimated date of delivery. At one facility, the respondent said village chiefs were enlisted to help ensure HIVpositive women were able to travel to the hospital for delivery. Only at one facility did the nurse say that the birth test is often missed if the woman delivers elsewhere. This is due to residing in a remote area with a poor network that makes follow-up via phone difficult.

\section{Retention}

Retention and treatment initiation addresses the potential influences of very early infant diagnosis on subsequent infant HIV testing and clinic attendance, reactions to a positive diagnosis and treatment initiation, and any differences between birth and other time points.

\section{Subsequent testing and clinic attendance}

Women and HWs did not indicate that birth testing affected subsequent infant HIV testing acceptance or clinic attendance. Those few HWs who encountered women who refused birth testing at their health facility indicated that women still accepted subsequent infant tests and that the birth DNA-PCR test was the only issue, reasoning that the underlying cause was insufficient sensitization, since birth testing was only in a pilot phase. One exception was described in which a woman's child received a birth test (with a negative result), but did not return at 6 or 14 weeks because the woman did not want to take ART for herself and was avoiding the clinic. HWs felt that clinic attendance would either not be impacted by the timing of infant diagnosis or that women who have infants infected at birth would have better attendance, given that they would be more highly motivated and need to return regularly for refills and more frequent monitoring. Women did not indicate that the addition of a birth test negatively influenced their motivations to attend standard clinic services, citing the necessary postpartum care needed (e.g., immunizations, child examinations, drug refills).

\section{Very early infant diagnosis and treatment initiation}

Nurses were responsible for actually initiating infants on ART, but HWs indicated that all staff were responsible for ensuring that they were initiated on treatment as soon as possible (while waiting for confirmatory testing), for infants infected at any age. No respondents expressed a challenge with early initiation, but only the perspective of HWs was captured, as all women interviewed had HIV-negative children. HWs described typical reactions of mother and caregivers 
upon learning their child was HIV-positive, such as guilt, sadness, and confusion. Nonetheless, they reported mothers were consoled by the opportunity to save their child's life through treatment, though intensive counseling was often needed to help them understand the diagnosis and implications of treatment. Reactions were not felt to vary between mothers learning their child was infected at birth versus other time points; counseling and individual personalities were more relevant with regards to treatment acceptability for children. The only difference that HWs could foresee was that mothers would experience greater guilt if their child was found to be infected at six weeks versus birth, since they felt mothers perceived more agency over the health of their child after delivery.

\section{DATA ABSTRACTION FINDINGS: DIAGNOSIS AND TREATMENT TURNAROUND TIMES}

Overall, all HIV-positive women enrolled in the PEA-WIL study delivered as of October 2016. Of these 614 HIV-exposed infants born to 606 women, a birth test was not performed for 29 percent of them ( $n=178$ ). Nine mothers (1.5 percent) declined birth testing and 22 infants (4 percent) died within the first hours or days of life. Other reasons included home delivery, delivery over a weekend or other time without a study nurse on duty, delivery at a non-study facility, and failure to perform the test by a study nurse. Only one was due to a test kit stock-out and one other was because the nurse failed to obtain a sufficient specimen. When birth specimens were collected, they were packaged and transferred according to the standard EID procedures described in the above section.

The results in this section are presented by the two sub-activities as described in the methods.

\section{Diagnosis TAT}

One sub-activity was to calculate TAT for ART initiation for HIV-positive infants and other interim steps along the VEID pathway for HIV-exposed infants. Table 2 presents all of the HIV-exposed infants born between 12 January (following MOH IRB approval of the VEID study, received on 11 January) and 16 June 2016, to allow for approximately 6 months of data collection. The purpose of this exercise was to determine TAT for DNA-PCR tests conducted at birth and six weeks among study HIV-exposed and HIV-positive infants as well as compare the 6-week test TAT with non-study facilities. There were 50 PEA-WIL infants from eight study facilities meeting this criteria, including one identified as infected at 6 weeks, plus 1 infant identified as infected at birth (in another of the study facilities). There were 88 infants from 10 non PEA-WIL facilities; 5 children were identified as infected at 6 weeks, however, 1 child was excluded from the analysis as data on TAT could not be verified from source documentation. There were significant gaps in data availability, with more incomplete information at non-study facilities. 


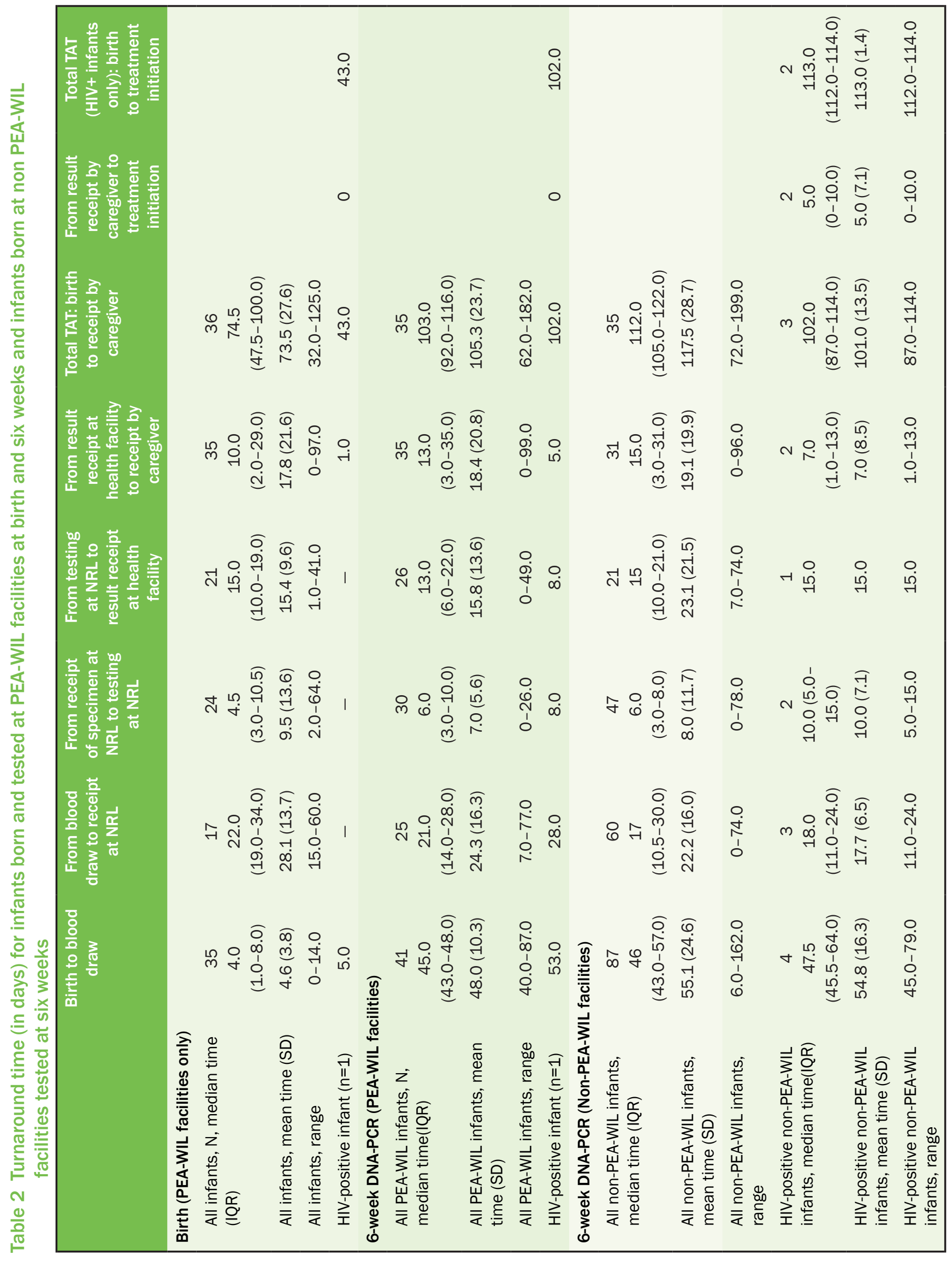


The data did not appear to be normally distributed; thus the median and interquartile range (IQR) are presented. However, as the mean and median are considerably different, both are included in the table. Median TAT from birth to caregiver result receipt was 74 days (IQR 47.5-100) for those infants tested at birth (36/50 infants had this information available). Median TAT from birth to caregiver result receipt was 103 days (IQR 92-116) for the PEA-WIL infants tested at 6 weeks $(n=35)$. Infants at non-PEA-WIL sites tested at 6 weeks with information available $(n=35 / 88)$ had a slightly longer median TAT of 112 days (IQR 105-122). There were few HIV-positive infants; the TAT from birth to ART initiation was 43 days for the 1 infant identified as infected at birth, the TAT for the 1 HIV-positive PEA-WIL infant identified at 6 weeks was 102 days, and there was a median of 113 days (IQR 112-114) for 2/4 infants identified as infected at 6 weeks from non-PEA-WIL facilities with information available. The time from HIV diagnosis (caregiver receipt of results) was the same day for the PEA-WIL infant identified as infected at birth, the PEA-WIL infant identified as infected at six weeks, and one non-PEA-WIL infant identified as infected at six weeks. The time was 10 days for the only other non-PEA-WIL infant with information available.

TAT was also constructed from time of blood draw to caregiver receipt of results or ART initiation to make a more direct comparison of TAT and to understand if the processes differed for birth versus 6-week testing. At PEA-WIL facilities, TAT to caregiver receipt of results was slightly longer at birth ( 69 days, IQR 47-95, $n=29$ ) versus 6 weeks (62.5 days, IQR 54-71.5, $n=28$ ) for all infants. At non-PEA-WIL facilities, median TAT from blood draw to caregiver receipt of 6-week DNAPCR results was 70 days (IQR 42-77, $n=35$ ). The TAT to ART initiation for the one PEA-WIL study child identified as infected at birth was 38 days. Children diagnosed at 6 weeks at PEA-WIL versus non-PEA-WIL facilities had similar TAT: 49 days for the 1 HIV-positive PEA-WIL study child and a median of 50.5 days (IQR 35-66) for the 2/4 non-PEA-WIL children.

Because this exercise yielded very few HIV-positive children contributing TAT outcomes, TAT were also calculated for all PEA-WIL positive children (as of October 2016) regardless of when they became infected (Appendix C). Median TAT was 55.5 days (IQR 40-139) for the 10/11 HIVpositive children with data available at blood draw and ART initiation. TAT from date of blood draw to ART initiation for the PEA-WIL study infants identified as infected at birth ranged from 35 to 62 days. Overall TAT was 49 and 63 days for the 2 infants identified as infected at 6 weeks, 177 and 199 days for 2 out of the 3 infants identified as infected at 14 weeks, and 40 and 139 days for the 2 infants identified as infected at 6 months.

\section{Treatment TAT}

Another sub-activity was to review demographic and clinical profiles of all women with positive children from the PEA-WIL and Kabeho cohorts and calculate TAT for all HIV-positive children diagnosed by DNA-PCR testing in Lesotho.

Appendix D presents selected demographic and clinical characteristics of HIV-positive women and their positive infants in Lesotho $(n=11)$. Three HIV-positive infants died-two from respiratory infections and one from malnutrition. One of these infants was diagnosed with HIV from birth testing, initiated on ART at 6 weeks, and died at approximately 14 weeks of age from a respiratory infection. In total, 8 out of the 11 women were not on ART at first ANC visit, 3 of whom had a child infected at birth. Of these 8, their gestational age at first ANC visit ranged from 4 to 32 weeks. 
Eight of the women ( 3 with children identified as infected at birth) had maternal viral load results at study enrollment in ANC, all of which were $>1,000$ copies $/ \mathrm{ml}$.

Appendix E presents selected demographic and clinical characteristics of HIV-positive women and their positive infants in Rwanda. 92 percent ( $n=545 / 595)$ of live born infants, excluding those who died within 2 hours of birth, had blood drawn and stored for a birth test. 95 percent $(n=517 / 545)$ had retrospective DNA-PCR testing performed. Two infants were infected at birth. Six out of 512 infants born to HIV-positive women who were still enrolled in the study by the end of the 24-month study follow-up period were diagnosed as HIV-positive. All six HIV-positive children in Rwanda were initiated on treatment and none of the six HIV-positive children died. Those women whose children were infected at birth had the shortest duration between study enrollment and HIV diagnosis and ART initiation. Four out of the 5 mothers with a viral load test at study enrollment had a result of $>1,000$ copies $/ \mathrm{ml}$, including those with the 2 infants with infection present at birth.

\section{COSTS ASSOCIATED WITH VEID}

In 2015, 199 infants received a VEID test from the 8 facilities included in the VEID costing exercise in Lesotho, while 287 VEID tests were performed at the NRL. (The PEA-WIL study included five other facilities that were performing birth testing, but were not included in the costing assessment.) Test results indicated that only one out of 199 ( 0.5 percent) VEID costing study infants was found to be HIV-positive on birth testing and was initiated on antiretroviral treatment.

The unit cost of VEID services (including counseling, drawing, packing and transporting samples, and performing the test at the NRL) was determined to be $\$ 40.50$. Major cost drivers were supplies and commodities (46 percent), clinical labor (22 percent), construction cost/rental value of the facilities (17 percent), sample transportation cost (11 percent), support staff (3 percent), and equipment (1 percent).

\section{Lesotho}

In order to assess the potential impact of nationally scaling up VEID in Lesotho, the following additional assessment was performed. In 2015, 11,744 children were born to HIV-positive mothers in Lesotho and approximately 11,157 births (assuming no multiple births) were covered by Lesotho's PMTCT program intervention. At the 8 PEA-WIL study facilities that were included in the costing analysis, 66 percent (199/300) of PEA-WIL infants born at these facilities in 2015 received a VEID test. Reasons for non-performance in the remaining 34 percent were identified in the preceding section. If we assume that at minimum, a national program might test a similar proportion of HIV-exposed infants at birth, then nationally 7,401 HIV-exposed children would receive birth testing. Out of the 199 infants, 1 was found HIV-positive and initiated on treatment (0.5 percent in utero infection rate).Thus, using a conservative estimate based on 66 percent coverage nationally with VEID and an in utero infection rate of 0.5 percent, 37 HIV-positive infants would have been identified as infected at birth and benefited from this service if VEID was offered nationally in 2015, and the total incremental cost of adding a VEID test at birth would 
have been $\$ 299,618$ ( $\$ 40.50$ per recipient times 7,401 children tested at birth). Thus dividing the incremental cost by the number of beneficiaries (37) yields $\$ 8,057$ as the cost per child benefiting from VEID. This same result is obtained by simply multiplying the VEID unit cost by the total number of infants who received the test during the pilot phase ( $\$ 40.50$ times 199). Using an estimate of 21 percent ${ }^{2}$ mortality by age two months in children with peripartum infection without antiretroviral therapy (Becquet 2012), a national VEID program with immediate initiation of ART would theoretically have averted eight early deaths among infants.

\section{Rwanda}

VEID testing was assessed retrospectively in Rwanda using 2013/2014 data. Out of 595 HIVexposed newborn infants, 517 VEID samples, representing 87 percent $(517 / 595)$ of infants, were successfully processed, and two ( 0.4 percent) infants were found to be HIV-positive at birth. National data from 2011-2015 were used to project the expected impact VEID could have had on pediatric HIV in Rwanda. Using 87 percent VEID national coverage estimated from coverage from the Rwanda Kabeho study and 0.4 percent in utero HIV infection rate, 22 HIV-positive infants would have been identified as infected at birth and benefited from this intervention, which theoretically could have averted five early deaths if VEID if immediate initiation of treatment was offered nationally during the study period. Since the estimation of the unit cost of providing VEID in Lesotho by this study is the first of its kind, this information was used to project the expected cost per child benefiting from VEID in Rwanda during the study period, which would have been $\$ 10,381$.

\section{Sensitivity Analysis}

Considering that some of the reasons that VEID tests were not conducted were due to birth testing implementation only as a part of the PEA-WIL study (including delivery at non-study facility and absence of a study nurse at the facility), it is uncertain what the coverage would be if this service was scaled up nationally. For this reason, VEID coverage varied from a pessimistic value of 50 percent to a very ambitious target of 100 percent coverage. The rate of in utero HIV infection also varied from 0.5 to 5 percent in Lesotho. The rate of in utero infection in the Rwanda Kabeho study was lower than in Lesotho ( 0.4 percent versus 0.5 percent); thus the rate of in utero infection for this exercise varied from 0.4 to 3 percent.

Based on the estimated number of births to HIV-positive women in 2015 in Lesotho and Rwanda, Tables 3 and 4 provide costs outcomes of various scenarios where VEID coverage and the percentage of infants diagnosed HIV-positive at birth were varied as noted above. It is important to note that the rate of in utero infection does not affect the cost of diagnosis (VEID unit cost), but does affect the cost per child identified. Thus, as the rate of in utero infection increases, the cost per HIV-positive child identified at birth decreases. Also, VEID coverage impacts both the number of infants benefiting from the intervention as well as the number of early infant deaths averted, but not the cost per infant benefiting from VEID (Tables 3 and 4 ).

\footnotetext{
${ }^{2} 18$-month post-infection mortality risks in the absence of treatment is about 60 percent, which translates to a conservative estimate of about 21 percent in the first two months of life.
} 
Table 3 Cost outcome of VEID in Lesotho in 2015

\begin{tabular}{|c|c|c|c|c|c|c|c|}
\hline $\begin{array}{l}\text { VEID } \\
\text { coverage }\end{array}$ & $\begin{array}{l}\% \text { of infants diagnosed } \\
\text { HIV+ at birth }\end{array}$ & $0.5 \%$ & $1 \%$ & $2 \%$ & $3 \%$ & $4 \%$ & $5 \%$ \\
\hline \multirow{2}{*}{$50 \%$} & Deaths averted & 6 & 12 & 23 & 35 & 47 & 59 \\
\hline & Infants benefiting from VEID & 28 & 56 & 112 & 167 & 223 & 279 \\
\hline \multirow{2}{*}{$60 \%$} & Deaths averted & 7 & 14 & 28 & 42 & 56 & 70 \\
\hline & Infants benefiting from VEID & 34 & 67 & 134 & 201 & 268 & 335 \\
\hline \multirow{2}{*}{$63.3 \%$} & Deaths averted & 8 & 16 & 31 & 47 & 62 & 78 \\
\hline & Infants benefiting from VEID & 37 & 74 & 148 & 222 & 296 & 370 \\
\hline \multirow{2}{*}{$70 \%$} & Deaths averted & 8 & 16 & 33 & 49 & 66 & 82 \\
\hline & Infants benefiting from VEID & 39 & 78 & 156 & 234 & 312 & 390 \\
\hline \multirow{2}{*}{$80 \%$} & Deaths averted & 9 & 19 & 37 & 56 & 75 & 94 \\
\hline & Infants benefiting from VEID & 45 & 89 & 179 & 268 & 357 & 446 \\
\hline \multirow{2}{*}{$90 \%$} & Deaths averted & 11 & 21 & 42 & 63 & 84 & 105 \\
\hline & Infants benefiting from VEID & 50 & 100 & 201 & 301 & 402 & 502 \\
\hline \multirow{3}{*}{$100 \%$} & Deaths averted & 12 & 23 & 47 & 70 & 94 & 117 \\
\hline & Infants benefiting from VEID & 56 & 112 & 223 & 335 & 446 & 558 \\
\hline & $\begin{array}{l}\text { Cost per infant benefiting } \\
\text { from VEID }\end{array}$ & $\$ 8,057$ & $\$ 4,049$ & $\$ 2,024$ & $\$ 1,350$ & $\$ 1,012$ & $\$ 810$ \\
\hline
\end{tabular}

Table 4 Cost outcome of VEID in Rwanda in 2015

\begin{tabular}{|c|c|c|c|c|c|c|c|}
\hline $\begin{array}{l}\text { VEID } \\
\text { coverage }\end{array}$ & $\begin{array}{c}\% \text { of infants diagnosed } \\
\text { HIV+ at birth }\end{array}$ & $0.4 \%$ & $1 \%$ & $1.5 \%$ & $2 \%$ & $2.5 \%$ & $3 \%$ \\
\hline \multirow{2}{*}{$50 \%$} & Deaths averted & 3 & 7 & 10 & 14 & 17 & 21 \\
\hline & Infants benefiting from VEID & 13 & 33 & 50 & 66 & 83 & 99 \\
\hline \multirow{2}{*}{$60 \%$} & Deaths averted & 3 & 8 & 12 & 17 & 21 & 25 \\
\hline & Infants benefiting from VEID & 15 & 40 & 60 & 79 & 99 & 119 \\
\hline \multirow{2}{*}{$70 \%$} & Deaths averted & 4 & 10 & 15 & 19 & 24 & 29 \\
\hline & Infants benefiting from VEID & 18 & 46 & 69 & 93 & 116 & 139 \\
\hline \multirow{2}{*}{$80 \%$} & Deaths averted & 4 & 11 & 17 & 22 & 28 & 33 \\
\hline & Infants benefiting from VEID & 20 & 53 & 79 & 106 & 132 & 159 \\
\hline \multirow{2}{*}{$87 \%$} & Deaths averted & 5 & 12 & 18 & 24 & 30 & 36 \\
\hline & Infants benefiting from VEID & 22 & 57 & 86 & 115 & 144 & 173 \\
\hline \multirow{2}{*}{$90 \%$} & Deaths averted & 5 & 12 & 19 & 25 & 31 & 37 \\
\hline & Infants benefiting from VEID & 23 & 60 & 89 & 119 & 149 & 179 \\
\hline \multirow{3}{*}{$100 \%$} & Deaths averted & 5 & 14 & 21 & 28 & 35 & 42 \\
\hline & Infants benefiting from VEID & 26 & 66 & 99 & 132 & 165 & 198 \\
\hline & $\begin{array}{l}\text { Cost per infant benefiting from } \\
\text { VEID }\end{array}$ & $\$ 10,465$ & $\$ 4,049$ & $\$ 2,699$ & $\$ 2,024$ & $\$ 1,619$ & $\$ 1,350$ \\
\hline
\end{tabular}


Sensitivity analysis results indicated that VEID cost per HIV-positive child identified at birth is lower when the risk of in utero infection is high. Since the observed rate of in utero infection in the Rwanda Kabeho study (0.4 percent) was lower than in the Lesotho PEA-WIL study ( 0.5 percent), using these infection rates, the estimated cost per HIV-positive child was higher in Rwanda than Lesotho ( $\$ 10,465$ versus $\$ 8,057$, respectively). However, should the in utero infection rate be the same in the two countries, the cost per HIV-positive infant identified would be the same (i.e., if the in utero infection rate was 1 percent in both countries, the cost per child benefitting would be $\$ 4,049$ in both countries). 


\section{DISCUSSION}

Overall, birth testing was acceptable to respondents participating in the in-depth interviews, with most recommending the roll out of birth testing nationally and universally and nearly all women reporting they were happy to have their child tested or to know their child's HIV status at birth. However, the feasibility of the approach was called into question. Many HWs and lab respondents identified challenges reflective of additional burdens to the larger shortcomings with the EID system, such as workload and reagent stock-outs, particularly at the NRL, poor communication between facilities and laboratories, and delays in the return of HIV test results; women also raised the latter as a concern. Some challenges were also due simply to the pilot nature of birth testing, which meant procedures were not routinized and there was limited involvement from clinic staff. VEID-specific challenges included concern regarding obtaining blood from a newly delivered child, which was noted rarely, some concerns from health workers and lab staff over obtaining a sufficient and quality sample, and the potential for missed birth testing opportunities when women deliver at home. What appears most important for birth testing to be successful are strengthened follow-up systems for women not delivering at a health facility, early and intensive counseling so women understand the continuum of DNA-PCR testing, and improvements to steps along the EID pathway, specifically concerning specimen transfer and delivery of results.

One of the main challenges with birth testing was ensuring that a child was tested at birth or as soon as possible thereafter. While the pilot nature of birth testing limited the number of children who were tested, since birth testing only took place in study facilities and primarily only was performed by study nurses, this experience does offer some lessons learned for what activities should accompany a national rollout. This includes updating group counseling messages that HIV testing will begin at birth and not six weeks and to emphasize the importance of mother-infant pairs' attendance at the seven-day visit, particularly if women deliver at home or in South Africa rather than Lesotho. Awareness-raising outside the clinic settings so that HIV-positive women will anticipate an infant birth test may also contribute to acceptance of early testing.

Given that the Lesotho Demographic and Health Survey found that nearly a quarter of women delivered at home (Lesotho $\mathrm{MOH}$ 2016), the potential for missing the birth test window is considerable. Having a standard 7-day postnatal visit in Lesotho helped to capture women whose infants could not be tested at delivery, even though HWs admitted this visit was not adhered to as well as the six-week visit. While attendance may still be limited due to the proximity of the visit to delivery, adding a DNA-PCR test by two weeks of age (the definition of birth testing used in this study) to the algorithm could improve adherence to this visit. The importance of this visit to capture some birth tests which may have been missed otherwise also raises the question of what happens in other countries in which a 7-day visit is not standard.

Some of the women interviewed perceived counseling on HIV birth testing to be limited. There was also a disconnect between the counseling descriptions provided by women and the more in-depth ones provided by health workers interviewed, which could be recall bias or reflect shortcomings with the counseling itself. Women also noted that counseling on subsequent HIV 
tests was minimal. What was similar across both participant groups was that counseling on HIV birth testing took place at ANC visits as well as around the time of delivery. This was likely due to the fact that PEA-WIL study women had already given consent and birth testing was included as one of the activities about which they were informed before they agreed to join the study. HWs also said that women may have been concerned about birth testing or infant HIV testing in general, but that intensive counseling was needed for some women to understand its importance. As above, counseling on birth testing, how it fits into the EID testing algorithm and the importance of all DNA-PCR testing needs to be conveyed at every opportunity with women, potentially starting in the community or at the time of ANC group education.

As expected, the TAT from birth to caregiver receipt of results was shorter for PEA-WIL infants tested at birth versus 6 weeks (74 vs. 103 days). Among the HIV-positive PEA-WIL infants, the TAT from birth to ART initiation was also shorter for the infant infected at birth versus 6 weeks (43 vs. 102 days).

When comparing TAT from blood draw to caregiver receipt of results between birth and subsequent tests, a clear picture does not emerge. Among PEA-WIL infants born between January and June 2016, TAT to caregiver receipt of results was slightly longer at birth (67.5 days) versus six weeks (62.5 days). When examining only PEA-WIL positive infants, there is a somewhat linear trend toward increased TAT with increasing child age. However, all HWs and laboratory staff reported that the DNA-PCR processing, transporting, and testing processes were the same at birth and six weeks, and this TAT only captures two infants. This may be related to fewer visits to the clinic for immunizations and $\mathrm{MCH}$ follow-up at later ages. TAT from blood draw to caregiver receipt of results was a week longer at non-PEA-WIL facilities among all infants; when comparing the HIV-positive infants' time to treatment initiation, these were fairly similar. This suggests PEAWIL infants may have benefited from the extra support afforded in the study, but only slightly.

There was evidence of fast-tracking HIV-positive results, with shorter TAT from blood draw to caregiver receipt of results: 55.5 days for HIV-positive infants at all time points versus 67.5 days and 62.5 for birth and 6 weeks tests, respectively. This is consistent with the qualitative findings, which describe some fast-tracking mechanisms, though not necessarily standardized or formalized. All four infants infected at birth initiated ART within six to nine weeks of age, likely at their six week visit. However, because the maximum TAT to caregiver receipt of results was 125 days (approximately 16-18 weeks from the time of the birth test), there would have been children having blood drawn again at subsequent visits without yet having received birth test results.

Differences aside, TAT was still found to be long and largely unchanged from the study conducted on TAT in Lesotho five years ago (Gill et al. 2013). While the respondents interviewed at the clinic, district lab, and central lab level all have clearly defined roles in the EID pathway, they are distinct and separate from each other, to the point that respondents could really only speak about their responsibilities and supposed the bottlenecks were happening at another level. The most effective solution to the transport challenges identified may be point-of-care machines to bring EID services closer to clients. Rollout of five point-of-care HIV testing machines supported by UNITAID in Lesotho (including one study district) is anticipated for the end of 2016 (EGPAF communication). Since the transportation of samples and some of the related challenges are unavoidable, other potential solutions could involve more efficient ways of delivering results- 
through a functional LIS system between central and district labs, repairing and putting back into use SMS printers, or changes to the current communication flow that would allow for central and district lab staff to initiate communication of results to facilities.

Different strategies (no EID, testing once at birth, once at six weeks, and testing twice at birth and six weeks) were modeled as part of a study in South Africa (Francke et al. 2016). For their base case scenario, they used a lower cost per test than we identified through the costing exercise in Lesotho ( $\$ 25$ compared to $\$ 40.50$, respectively) and projected a 1.8 percent in utero infection rate (compared to the 0.5 percent we observed in PEA-WIL in Lesotho). They reported that EID testing twice at birth and six weeks improved outcomes and was cost-effective compared to EID at six weeks alone in South Africa. The incremental cost-effectiveness ratio (ICER) was $\$ 2,900$ / year of life saved, 45 percent of South Africa's per capita gross domestic product (GDP) (an ICER of $<50$ percent of GDP is considered cost-effective). The ICER became $>50$ percent GDP if: ARTassociated reductions in mortality/OI risk was $<78$ percent, test specificity was $<98$ percent or sensitivity $<30$ percent, linkage to ART was $<15$ percent, result-return was $<16$ percent, PMTCT coverage was $>97$ percent, test costs exceeded $\$ 31$, mean TAT was $>3$ months or clinical care costs were $>1.3$ times base values. Given $>95$ percent PMTCT coverage in Lesotho with an in utero transmission rate of 0.5 percent and a per test cost of $\$ 40.50$, testing at birth and 6 weeks may not be cost-effective in the Lesotho context. In this situation, strengthening the 6-week testing system and improving TAT is another viable option for improving outcomes for HIV-positive infants.

Based on various scenarios, VEID followed by immediate ART could annually avert between 8 and 117 early infant deaths in Lesotho, and between 7 and 69 in Rwanda, with the cost per child benefiting from VEID ranging from $\$ 810$ to $\$ 8,057$. The percentage of children who are identified as HIV-positive at birth does not affect the cost of diagnosis, but does affect the cost per HIVpositive child identified with VEID. Thus, as the in utero infection rate among newborns increases, the cost per HIV-positive child identified decreases. Also, VEID coverage impacts the number of infants benefiting from the intervention as well as the number of early infant deaths averted, but not on the cost per HIV-positive infant benefiting from VEID.

\section{LIMITATIONS}

This study has some limitations which are noted below. More details on the costing component limitations are provided in Appendix F.

1. Semi-structured interviews: Only a small number of women were interviewed on their infant HIV birth testing perspectives and no women who had a child infected at birth were interviewed, despite efforts to include them. Also, no interviews were conducted with clinic (non-study) nurses. These nurses were not involved in VEID as it was only in the pilot phase, though they may have viewed VEID less favorably. While they would certainly be involved if VEID were rolled out nationally, clinic counselors, who were interviewed as part of this study, would be primarily responsible for performing infant HIV counseling and testing.

2. Data abstraction: This exercise yielded relatively few HIV-positive infants diagnosed at different time points, making it difficult to discern whether the variability observed is actual 
or due to the small sample size. Also, some of these data were abstracted from existing facility records which were at times incomplete and could not always be linked to the national EID database.

\section{Costing:}

a. Personnel: Average salary was applied to the corresponding personnel category in all the study sites.

b. Effects of recall bias: No time-motion study was carried out to assess how staff spent their time on VEID activities.

c. VEID unit cost: VEID unit cost from Lesotho may not necessarily be representative of the unit cost in Rwanda. 


\section{CONCLUSION AND RECOMMENDATIONS}

\section{CONCLUSIONS}

Based on the qualitative findings, women, health workers, and laboratory personnel were in favor of expanding EID to birth, recognizing the importance of identifying and treating HIV-positive infants as early as possible. However, respondents also clearly outlined a number of challenges with the overall EID system, many of which were not specific to birth testing but would be exacerbated by the burden of an additional birth testing time point. These included stock-outs, lab staffing shortages, and unreliable or infrequent transportation of samples and results. These challenges help to explain the TAT of nearly two months for PEA-WIL children tested at all ages in the study thus far. Children diagnosed with HIV at birth were getting on treatment earlier than under the standard testing algorithm, but still not as quickly as hoped. If there was a stronger system in place a more compelling case could be made for birth testing.

It is beyond the scope of this study, given the small numbers of HIV-positive children and relatively few facilities implementing birth testing, to make a recommendation regarding birth testing for Lesotho or other similar settings. While this study demonstrated acceptability, it does raise questions about feasibility, namely those related to TAT, but also missed opportunities to test at birth and limited follow-up systems to ensure infants are tested within the first two weeks of life. Counseling, tracing, and testing efforts could be better focused on strengthening infant diagnosis beginning at six weeks of life rather than the addition of birth testing. More national investment to get shorter TAT at six weeks would also likely be less burdensome on the clinic and laboratory network. For instance, the shortest step in the VEID/EID pathway at study and non-study facilities was from receipt of specimen at the NRL to testing at the NRL. If the specimen reached the NRL, a test would be performed relatively quickly. However, this study highlighted the challenge of transferring specimens and returning results to caregivers expeditiously. Respondents offered several recommendations comparatively less expensive than birth testing, such as ensuring SMS printers and the LIS system are functioning properly and establishing communication from the laboratory to the clinic, to minimize TAT and the potential for lost samples and results.

However, another factor to consider is the anticipated rollout of POC HIV diagnostic testing machines in Lesotho, Rwanda, and elsewhere in sub-Saharan Africa. The addition of these machines would help to address challenges related to transportation of samples and results (including lost samples), lab staff shortages by task shifting of testing, and the limited capacity of a small number of machines at central level. Point-of-care HIV diagnostic testing machines would likely make birth testing more feasible, though this may also be an expensive option. Moreover, in a hub and spoke model, in which lower level facilities ("spokes") send EID samples for processing at select hospitals (called "hubs"), there are still opportunities for delayed transfer of specimens or results. 
VEID resulted in 1 child out of 199 (0.5 percent) in 2015 found HIV-positive at birth in the Lesotho PEA-WIL study and initiated on treatment, while 2 of 517 (0.4 percent) were found HIV-positive at birth retrospectively in the Kabeho study in Rwanda. The unit cost of VEID was $\$ 40.50$. Because the rate of in utero infection was higher in Lesotho than Rwanda, the estimated cost for VEID per HIV-positive child identified was lower in Lesotho than in Rwanda, respectively $\$ 8,057$ and $\$ 10,381$. Sensitivity analysis was performed on the two key parameters driving these costs-VEID coverage and the rate of in utero HIV infection among newborns.

\section{RECOMMENDATIONS}

Should birth testing be implemented nationwide in Lesotho, findings from this study suggest that women, health workers, and laboratory personnel would find this policy change acceptable. They also indicate that subsequent infant HIV testing and postpartum clinic attendance would not be affected by adding birth testing to the diagnostic algorithm. However, this study illuminated some challenges with the feasibility of this approach, particularly with regards to the time from HIV testing to the return of test results and treatment initiation for HIV-positive infants. While infants infected at birth were initiated on treatment more quickly than those tested at six weeks, the turnaround time was still long and varied between one and two months. In order to ensure timely treatment initiation for HIV-positive infants and minimize associated morbidity and mortality, VEID costs, and burden to the health system, testing at a singular national laboratory should also be compared to the implications of implementing other strategies to minimize turnaround times, such as strengthening the current system of the initial infant test at six weeks.

Respondents were in favor of universal testing for HIV-exposed infants, rather than selective testing for those born to HIV-positive women considered high risk. A review of the demographic and clinical profiles of study women with HIV-positive children revealed that most women had high viral load at enrollment regardless of the timing of infant infection. However, Kabeho study women whose children were infected at birth were more likely to have shorter duration of ART prior to delivery. There was not a consistent pattern in terms of risk factors, but statistical significance could not be established due to small numbers. A more in-depth investigation should explore the association between birth infection rate and women's risk factors.

Results from this study indicate that provision of VEID nationally could have potentially averted eight early deaths with about 37 infants identified as HIV-positive at birth benefiting from early treatment in Lesotho, and five early deaths averted with 22 infants identified as HIV-positive at birth benefiting from early treatment in Rwanda during a one year study period. If VEID coverage increases, the number of infants benefiting from the service increases as well as the number of early infant deaths averted. The cost per child benefiting from VEID is driven by the rate of in utero HIV infection, and this could range from $\$ 810$ with 5 percent in utero infection rate to $\$ 8,057$ with 0.5 percent in utero infection rate in Lesotho. Thus, costs are lowest when the risk of in utero infection is high. In situations where PMTCT programs are not well-established and maternal ART use is low, and hence the risk of MTCT remains relatively high, universal birth testing might be considered. In situations where PMTCT programs are well-established and most women are on ART during or even before pregnancy, and hence risk of MTCT is low, a targeted testing program of infants at high risk of in utero infection (e.g., no maternal antiretroviral therapy or $<4$ weeks of 
treatment during pregnancy) might be a cost-effective way of implementing birth testing. However, a targeted program may be more complex to implement on a national scale, which could result in high-risk infants not being identified and hence missing birth testing; countries will need to consider both cost as well as implementation feasibility in designing national programs. 


\section{REFERENCES}

Becquet, Renaud et al. 2012. "Children who acquire HIV infection perinatally are at higher risk of early death than those acquiring infection through breast milk: a meta-analysis," PLosOne 7(2): e28510.

Bourne, David et al. 2009. "Emergence of a peak in early infant mortality due to HIV/AIDS in South Africa," AIDS 23(1): 101-6.

Campbell OM et al. 2016. "Family planning, antenatal and delivery care: cross-sectional survey evidence on levels of coverage and inequalities by public and private sector in 57 low and middleincome countries," Trop Med Int Health. 21(4): 486-503.

Francke, Jordan et al. 2016. "Clinical impact and cost-effectiveness of early infant HIV diagnosis in South Africa: Test timing and frequency,” J Infect Dis. 214(9): 1319-1328.

Gill, Michelle et al. 2013. "Towards getting more HIV-positive infants on lifesaving treatment: Assessing turnaround times for early infant diagnosis in Lesotho," oral presentation at the 7th International AIDS Conference, Kuala Lumpur, 30 June-3 July.

Innes, Steve et al. 2014. "Early severe HIV disease precedes early antiretroviral therapy: are we too late?" J Int AIDS Soc 17:188914.

International Monetary Fund (IMF) 2012. "Kingdom of Lesotho: Poverty Reduction Strategy PaperJoint Staff Advisory Note.” Washington, DC: IMF. Retrieved November 15, 2016 (https://www.imf. org/external/pubs/ft/scr/2012/cr12331.pdf).

Lesotho Ministry of Health and ICF International 2016. "Lesotho Demographic and Health Survey 2014." Maseru, Lesotho: Ministry of Health and ICF International. Retrieved November 15, 2016 (https://www.dhsprogram.com/pubs/pdf/FR309/FR309.pdf).

Lilian, Rivka et al. 2013. "Birth diagnosis of HIV infection in infants to reduce infant mortality and monitor for elimination of mother-to-child transmission," PIDJ 32(10): 1080-5.

Lilian, Rivka et al. 2014. "A mathematical model of evaluating the timing of early diagnostic testing in HIV-exposed infants in South Africa," JAIDS 67: 341-348.

Magder, Laurence et al. 2005. "Risk factors for in utero and intrapartum transmission of HIV," J Acquir Immune Defic Syndr 38(1): 87-95.

Marinda, Edmore et al. 2007. "Child mortality according to maternal and infant HIV status in Zimbabwe," Pediatr Infect Dis J 26(6): 519-26. 
Marston, Milly et al. 2011. "Net survival of perinatally and postnatally HIV-positive children: a pooled analysis of individual data from sub-Saharan Africa," Int J Epidemiol 40(2): 385-96.

Newell, Marie-Louise et al. 2004. "Mortality of infected and uninfected infants born to HIVinfected mothers in Africa: a pooled analysis," Lancet. 364(9441): 1236-43.

Riders for Health 2012. “Impact Report 2012.” Retrieved November 15, 2016 (http://www.geog. biz/wp-content/uploads/2015/03/Riders.pdf).

Rwanda Ministry of Health 2015. The 2014-15 Rwanda Demographic and Health Survey (RDHS). Kigali, Rwanda: National Institute of Statistics of Rwanda, Rwanda Biomedical Center and the Ministry of Health.

Smith, Sara-Jane et al. 2014. "Early infant diagnosis of HIV and fast initiation of anti-retroviral therapy in a rural African setting: how well are we doing?" Paediatr Int Child Health 34(3): 203-7.

UNAIDS 2016. "Prevention Gap Report." Geneva, Switzerland: UNAIDS. Retrieved November 15, 2016 (http://www.unaids.org/en/resources/documents/2016/prevention-gap).

UNAIDS 2011. "Global Plan toward Elimination of New HIV Infection among Children by 2015 and Keeping their Mothers Alive: 2011-2015. Geneva, Switzerland: UNAIDS. Retrieved November 15, 2016 (http://files.unaids.org/en/media/unaids/contentassets/documents/ unaidspublication/2011/20110609 JC2137 Global-Plan-Elimination-HIV-Children en.pdf).

Violari, Avy et al. 2008. "Early antiretroviral therapy and mortality among HIV-infected infants," N Engl J Med 359(21): 2233-44. 


\section{APPENDICES}




\section{APPENDIX A}

\section{ILLUSTRATIVE QUOTES BY THEMATIC AREA OF WOMEN, HEALTH CARE PROVIDERS, AND LABORATORY PERSONNEL WHO PARTICIPATED IN IN-DEPTH INTERVIEWS}

\begin{abstract}
Response
Acceptability

VEID benefits

Illustrative quote

...it's like, most of the time you will find that you're interested to know your child's status at birth when you are HIV-positive, you would like to know immediately after birth your child's status....and you will have a good feeling when your child gets tested because you will be able to know their HIV status.... (Woman)

I think it actually helps us in earlier identification of infected children rather than waiting for 6 weeks and then from 6 weeks waiting for results is going to take around 4 weeks, that is, when you are going to realize that the infant is infected. But with the birth DNA I think mostly at 6 weeks the results will be back. So I think we can actually initiate the child earlier than when we wait for 6 weeks. (Counselor, Hospital)
\end{abstract}

VEID benefits/ challenges

VEID challenges

Birth test refusals

Perspectives on national policy recommendations

...but birth testing they feel that their children are still very young to be punctured and for the blood to be drawn from them.... Sometimes they feel that it is unnecessary whereas some feel that it is necessary because during labor some of them are frightened, there is still that mindset that says there is a possibility that a child may get infected and that birth testing alleviates their fear, knowing that at least I will know the results of birth as soon as possible rather than waiting for 6 weeks. (Nurse, $\mathrm{HC}$ )

If I find that [results] are not available, but have to draw her blood again yet I still do not know her status, stresses me, wondering what the condition might be, yes! (Woman)

There are going to be more samples and that is going to affect turn-around time...because I think at national level there is going to be high workload and it means processing a lot of samples-we are going to get the results later than we get them now. (District lab)

This birth DNA-PCR, if it were a national thing, it will mean when we do health education, we would include it and we would also mobilize in the community...It would mean these mothers would know to come for a 7 day visit and would also mean that mothers would know that their children are tested at birth. (Nurse, Hospital)

[Workload] would be very very high but in return we would be getting something very big for the country, sort of a victory. So you know sometimes to work for something important like that is really beneficial. (District lab)

It's just that they need patience maybe, so ... when she refuses, you say no she is refusing and then you go... but if you talk to them and then try to tell them, and then try to show them the importance of doing DBS at birth, they understand at the end, they do. (Nurse, $\mathrm{HC}$ )

...what I realized is that most parents do not want to find their children infected so they want to do whatever it takes to...know the status of their children. (Counselor, Hospital)

...Because we always talk about the four prongs of HIV infection...If we say there could be HIV infection during labor, what are we doing about it, this is the perfect thing that we should do about it, we should test babies at birth. (Nurse, HC) 
Counseling on and understanding of birth testing
I think it was because even before she said anything, I told her that before I left home for testing I had already counseled myself. I told myself that this is my life and I have to do the right thing to live and in the case of my baby as well I knew that I was supposed to do the right thing. (Woman, 14week visit)

...labor comes with emotions as women suffer many illnesses, post-natal depression or anything so at that time we have to understand that it might not mean she actually [refuses] but she might need some rest [after delivery]. So what I do is that I explain thoroughly to mothers "you know this is what needs to happen, the positives and the negatives... but I understand if you need time for me to explain further," and after explaining, they gave me their go-ahead and not because I forced them, [but] because I still reassure them that "I respect your opinion, it is you who will give me the go ahead and if you say no, I cannot do it." (Nurse, HC)

Specimen transfer and processing and relaying results (TAT)

\section{Specimen transfer} and processing and relaying results (Fasttracking positive results)

Home delivery follow-up
Turnaround times for all the results are the same because sometimes you might even find out that you have drawn this DNA-PCR at birth and at 6 weeks and then you get the one for 6 weeks and the one for birth has not yet arrived. (Nurse, Hospital)

.... when it's beyond two months, we then develop a doubt whether we will ever get them. We call NRL and ask where the results are. Sometimes they say they have never received such, then I would not know what had happened, but we would have drawn them within two weeks or two months.... (Nurse, Hospital)

[When they reach the lab do you have any knowledge of how long they spend before going to the central lab?] I really don't know how long they take because we often find them still piled there when we bring other specimens. (Counselor, Hospital)

We get the results after I think a month, a month and 2 weeks, a month or so. I think if the results are positive we find ways of contacting the mother but if it's negative she will be told when coming to the next visit at the clinic... when it's positive if we have got the numbers we call, we also have people in the communities who track such people immediately, such people are brought to the facility so that the infants would be initiated. (Counselor, Hospital)

All people in $\mathrm{MCH}$ who saw the results that are positive, it's their responsibility to call that lady. (Nurse, Hospital)

We always have an appointment for an estimated date of delivery for them so if that date approaches then we call them just to inquire if they have delivered because not all of them deliver here so we need to call them just to be sure where they are. If they are in this facility then it means maternity will inform us if such a delivery is there. (Counselor, Hospital)

[Do they come here when they are 6 weeks only or 7 days?] ...sometimes they complain the place is too far, no mode of transport, they struggle truly. You may find that a woman after delivering at 7 days, they are not yet fit to travel such long distance, it is truly very far. (Counselor, Hospital) 


\section{Retention and treatment}

Subsequent testing and clinic attendance
It is because the first time she was tested the infection was not detected, so it might be visible when she is growing up, so it is important to keep testing her. (Woman, 6-week visit)

Well I think those children who were infected at birth, the caregivers usually ensure that they come for services regularly...but for those who actually might get HIV-positive results later... what I've realized is that...the caregivers don't always come because they know that the child only comes for a prophylaxis not a lifelong treatment. Yah, so they just tend to miss appointments because they don't have any particular service they come for except for prophylaxis. (Counselor, Hospital)

Very early Infant diagnosis and treatment initiation at an earlier stage they will say at least my child will get treatment early enough...as compared to knowing at a later stage when the child could even be at a worse stage. (Counselor, HC)
A person is going to be scared, confused, and is going to be angry. But the other hand...if they know

They will react the same way because at that time they will see the benefits of performing the test earlier if they were taught well enough the benefits of knowing the status earlier then I think it would help them know that even if the child if infected, there is still time, there is hope that the child can still live a healthy better life, the earlier it is detected, the earlier you can get treatment and live happily ever after. (Nurse, HC)

It does not matter if the child is infected at birth or at 6 weeks or even at 12 months, 12 years. The reaction is still going to be the same. (Nurse, Hospital)

I think the one at 6 weeks...should have a lot of guilt because I think she would think maybe I did something wrong, I did not do as they say, I should take care of the baby, as compared to that one at birth because that one at birth might think maybe something went wrong during pregnancy or at birth.... (Nurse, Hospital) 


\section{APPENDIX B}

SPECIMEN TRANSFER FROM FACILITIES AND TAT:

RESPONSES FROM HWS AND LABORATORY PERSONNEL

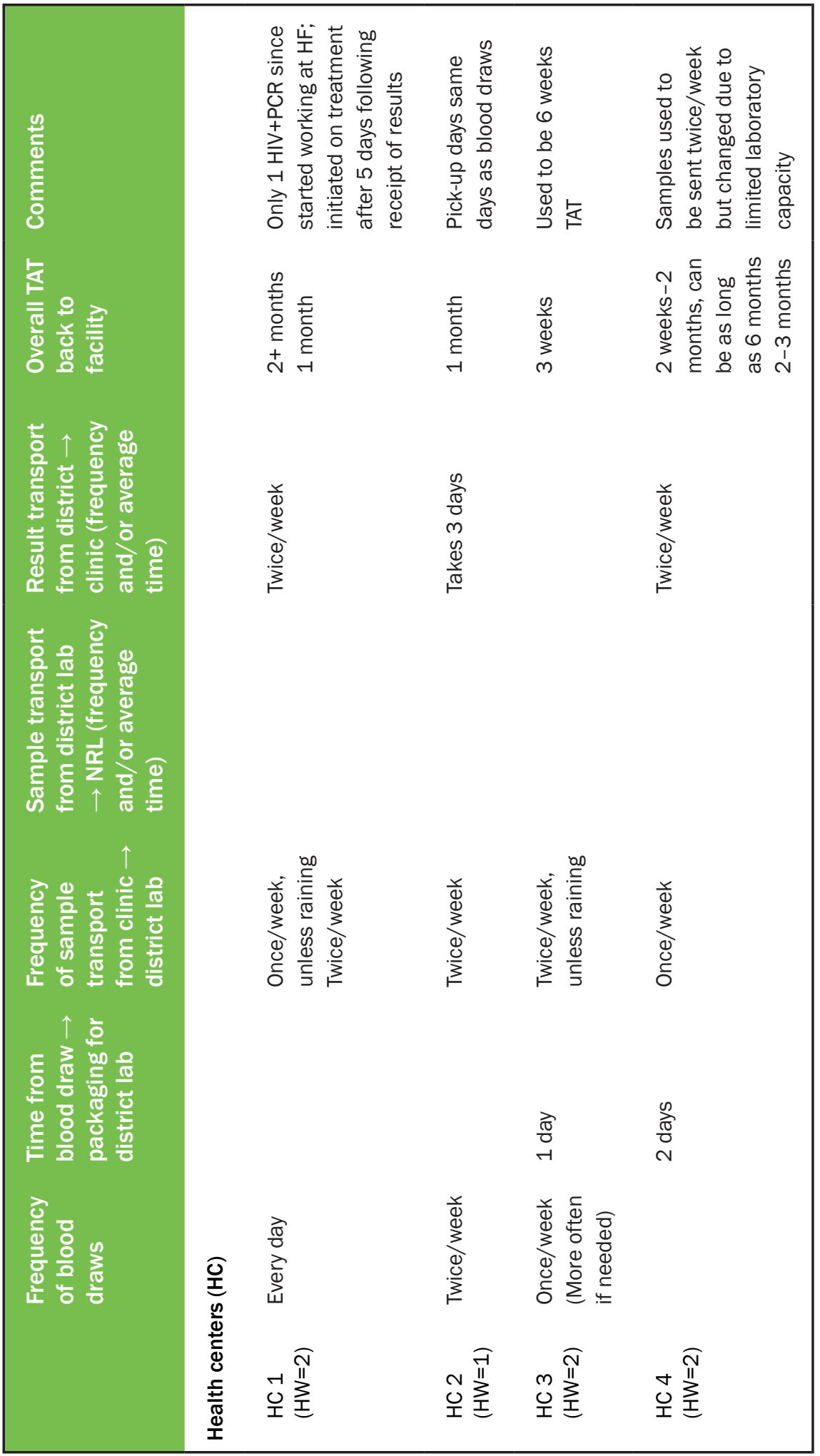




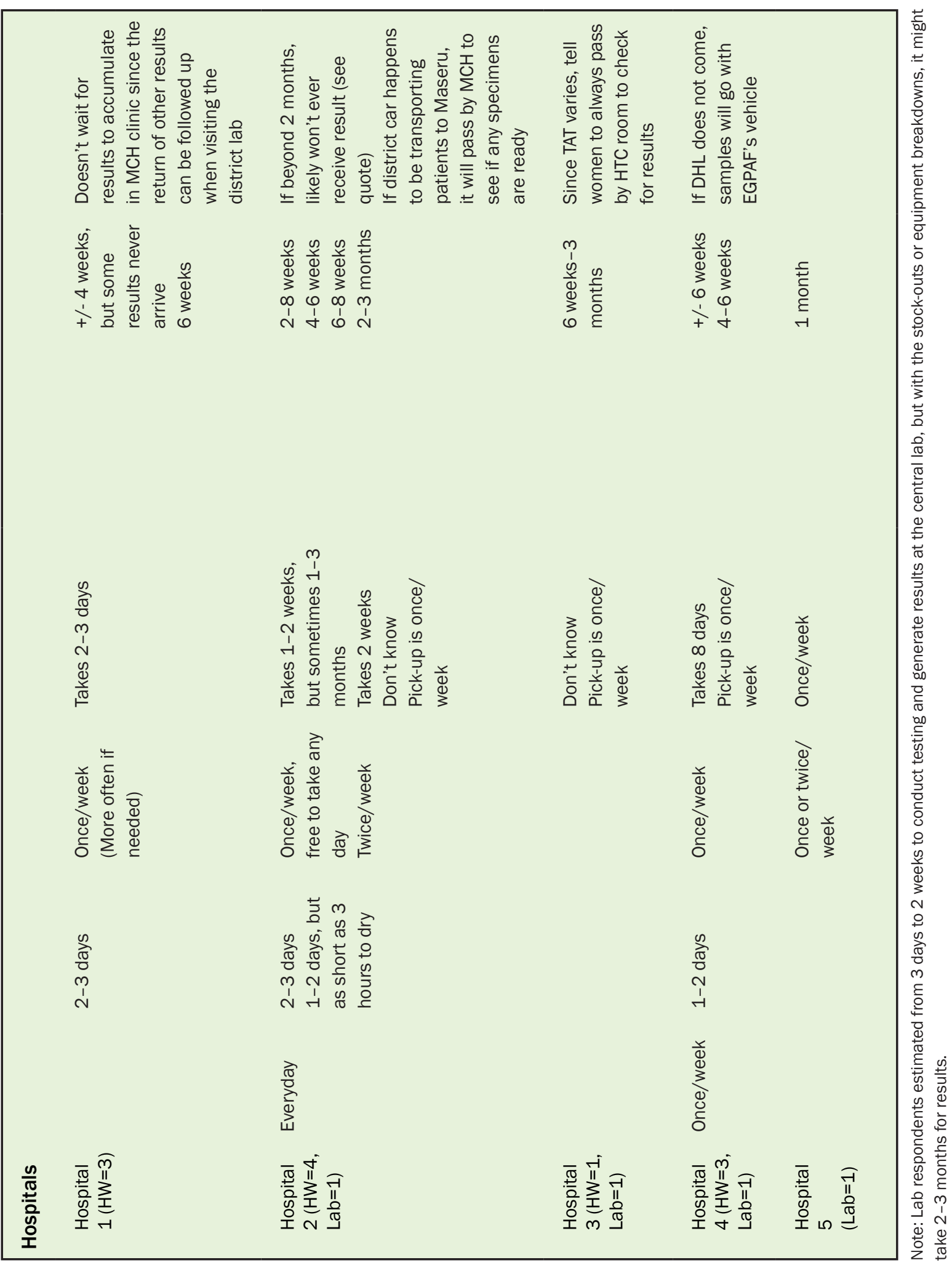




\section{APPENDIX C \\ TAT (IN DAYS) FOR PEA-WIL CHILDREN INFECTED WITH HIV SINCE START OF STUDY TESTED VIA DNA-PCR}

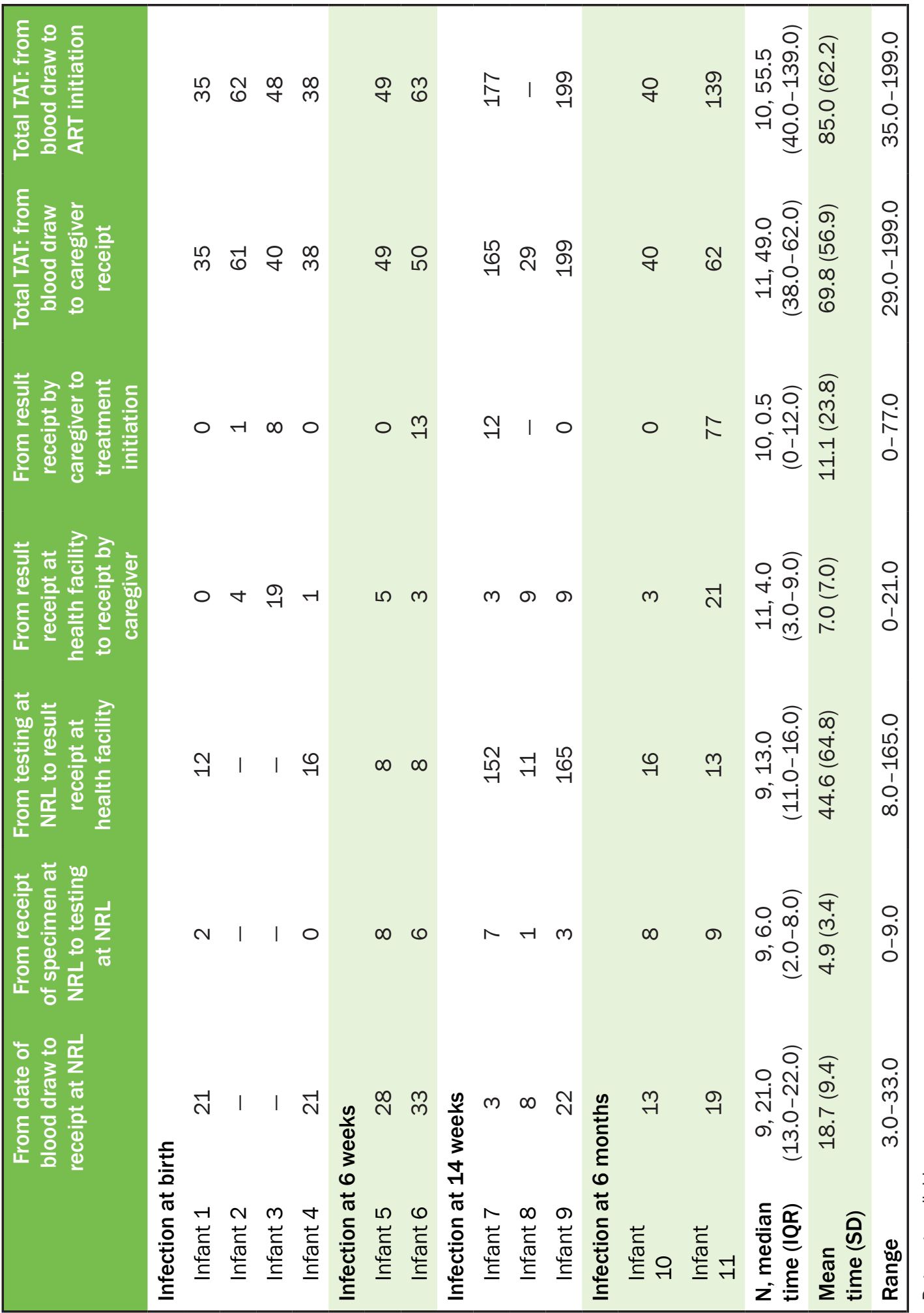




\section{APPENDIX D \\ SELECTED DEMOGRAPHIC AND CLINICAL CHARACTERISTICS \\ OF HIV-POSITIVE WOMEN AND THEIR INFECTED INFANTS IN LESOTHO ( $\mathrm{N}=11$ )}




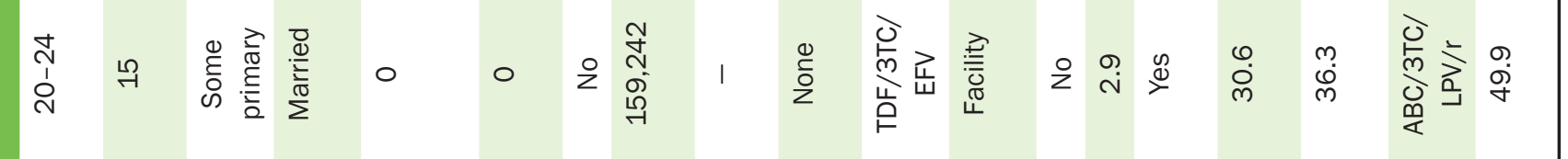

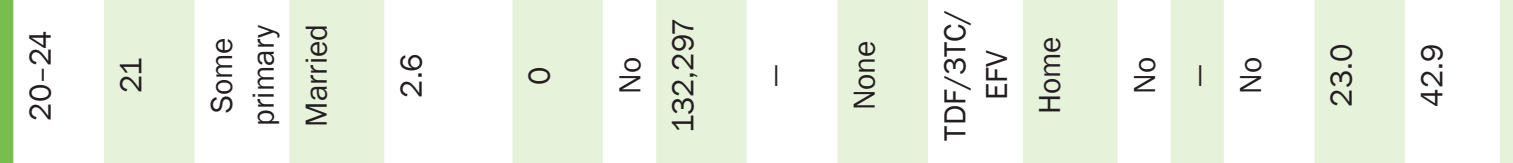

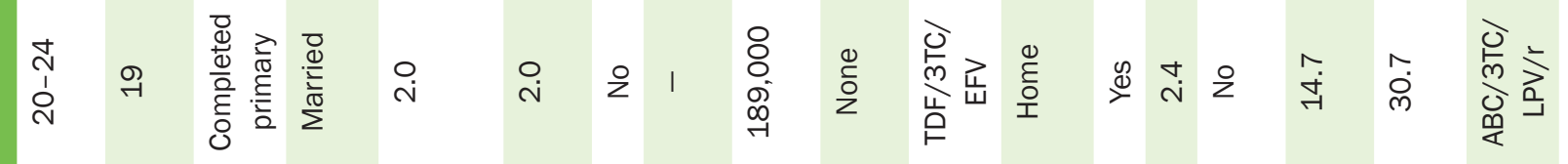

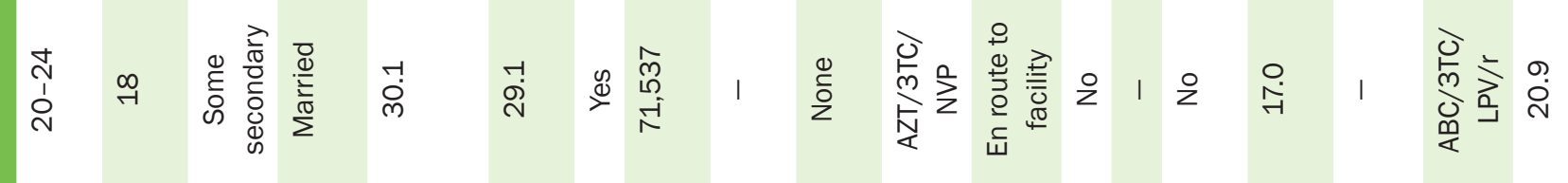
先

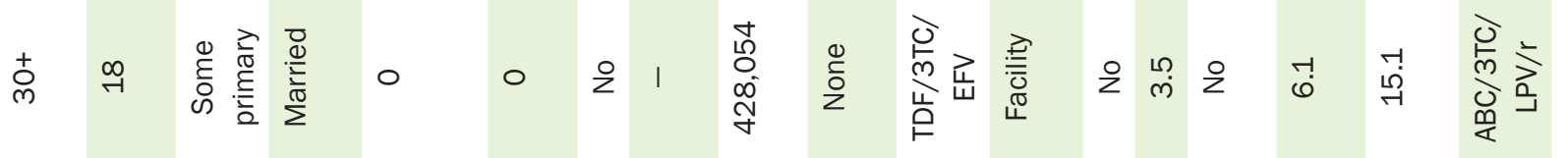

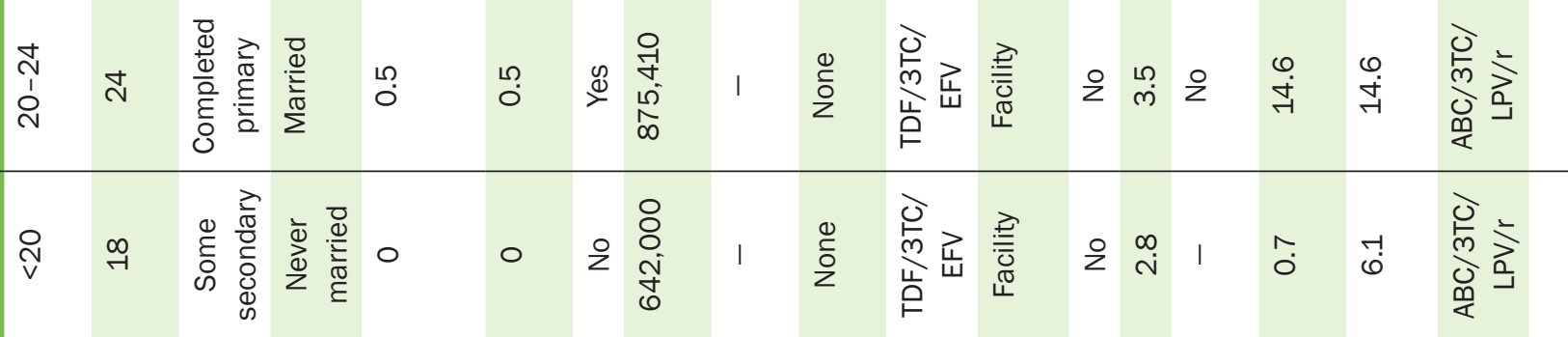

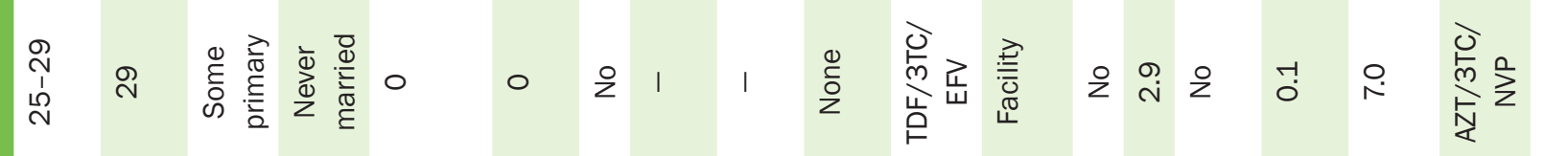

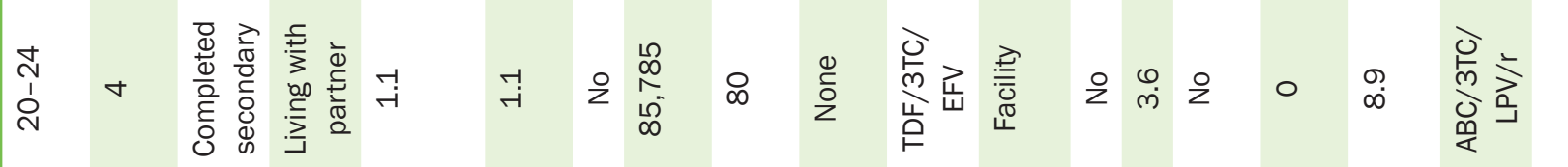

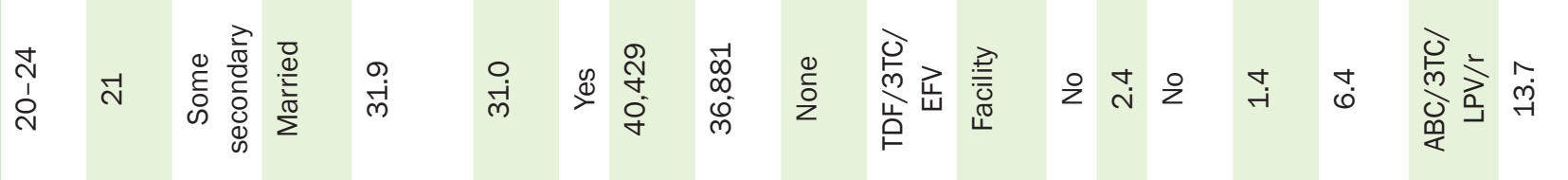

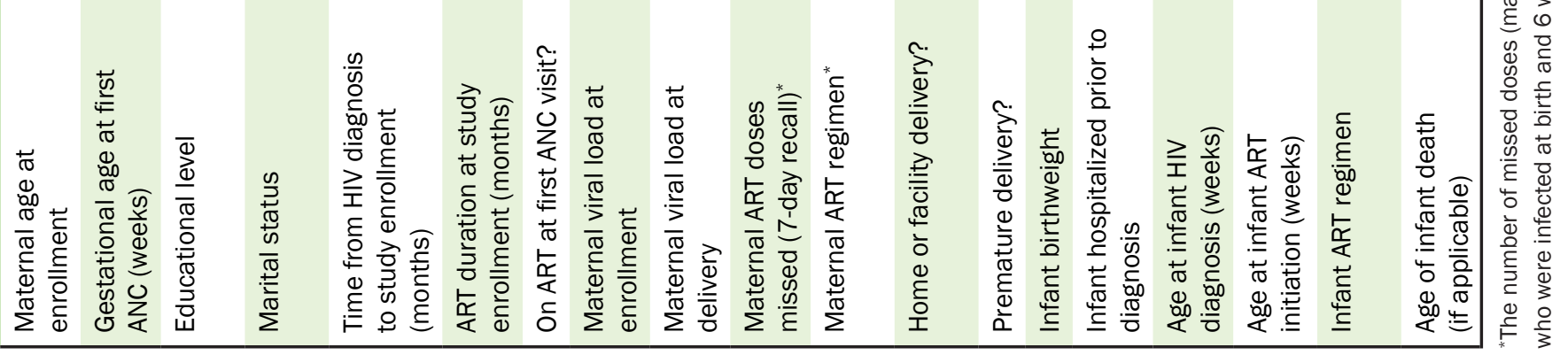




\section{APPENDIX E \\ SELECTED DEMOGRAPHIC AND CLINICAL CHARACTERISTICS \\ OF HIV-POSITIVE WOMEN AND THEIR INFECTED INFANTS IN \\ RWANDA ( $\mathbf{N}=6)$}

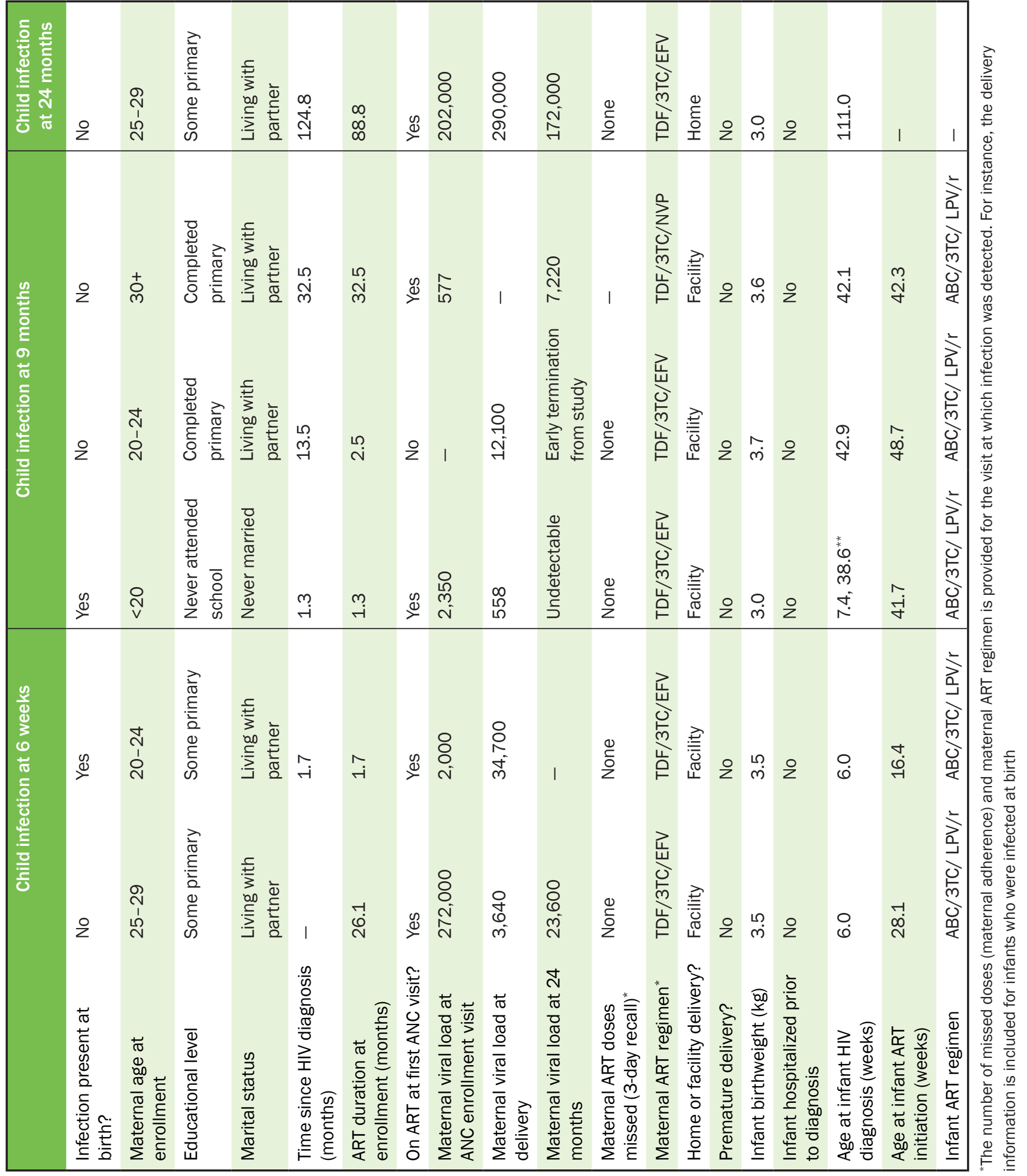




\section{APPENDIX F COSTING: FACILITY AND LABORATORY SURVEYS}

Facility and laboratory-based costing included interviews with key personnel at eight PEA-WIL sites across three EGPAF-supported districts in Lesotho: Thaba-Tseka, Butha-Buthe, Mohale's Hoek, and in Maseru where the NRL is located. At each facility, information was collected from clinical and support staff. Clinical or direct staff members comprised nurses, HTC counselors, and laboratory personnel. Support or indirect staff members included individuals who were generally not working directly on the VEID program. This included, for example, data clerks, accountants, maintenance staff, security guards, facility managers, office assistants, and receptionists.

Information on personnel employment status (permanent or contracted) was collected for both direct and indirect staff members. Additional information collected included the number of personnel, their salaries, and the level of effort or percentage of time expended on VEID services. The data indicated that no volunteer provides support to VEID services in the eight facilities surveyed.

\section{Equipment and Furniture}

Interviewees were asked to provide a list and count of all equipment and furniture utilized in the counseling area and laboratory or offices where the VEID samples are collected/processed. Facilities generally were not able to estimate the percentage of time that an item of equipment or furniture was used for VEID, and therefore the research team estimated this percentage based on the patient volume for VEID versus other services.

\section{Vehicles and VEID Samples Transportation}

VEID samples are transported by two courier services: Riders for Health (R4H) from health centers to district hospitals/labs-they also pick up results from district labs to health centersand by DHL from district facilities to the NRL and vice-versa. The costs of transporting VEID samples were obtained respectively from R4H annual report (Riders for Health 2012) and from the DHL office in Maseru. It is important to note that DHL provides transport services from the following district to the NRL (and collect results from the NRL to the district facilities): Thaba Tseka (St James Hospital), Butha Buthe (Butha Buthe and Seboche Hospitals), and Mohale's Hoek (Ntshekhe Hospital). Because these companies are contracted to transport EID samples, the cost of transporting EID or VEID samples is a fixed cost

\section{Utilities}

The costing of utilities was allocated based on the space used for VEID relative to the space of the entire facility. Costs related to the rental or construction value of the facility were also similarly apportioned to the EID and VEID program based on the number of patients seen. However, the $N R L$ construction value was not available, and thus it was assumed that the value of the facility was equivalent in rental terms to that of a median, "typical" facility in Lesotho. Because of limited data on property prices and rental market in Lesotho, the 2012 subsidized factory rental rates from the Lesotho National Development Corporation (LNDC) was used. In this case, the rental 
value of the facility was determined to be LSL41 per square meter (IMF 2012). This includes the average cost of rental (LSL8.5) and the average economic return to cover the initial capital costs and ongoing maintenance (LSL32.5).

\section{Cost Data}

Both direct (direct personnel, consumables) and indirect (indirect personnel, capital costs, maintenance and utilities) costs were collected at the eight study facilities as well as at the NRL. There was no cost associated with training to perform EID services because staff were already trained to perform EID services.

\section{Limitations}

- Personnel: Personnel (clinical and support staff) costs were often not available at the facility level. Robust efforts were made to obtain salary grades, with the average applied-to the corresponding personnel category-and across all the eight facilities; this approach could have potentially led to over- or under-estimation of both clinical and support staff wages.

- Effects of recall bias: No time-motion study was carried out to assess how staff spent their time on VEID activities. Instead, respondents were asked to provide their level of effort which could lead to effect of recall bias. The information obtained (which was based on recall and not on actual observations) was insufficient to be used for allocative purposes. Instead, an allocation method was developed, using the proportion of EID and VEID clients relative to the total client volume at the facility to apportion indirect staff time to VEID. It is therefore possible that there may have been an overestimation or underestimation of time spent on VEID in Lesotho.

- Utilities: Mootsinyane did not report any utility costs. Indeed, the following utilities were not available in some of the surveyed facilities:

- Water-James, St Theresa, St Peters, Tsepo, and Mootsinyane

- Electricity-St Theresa, St Peters, Butha Buthe, and Mootsinyane

- Construction year/rental value: Construction cost of the NRL was not available and there were limited data on property prices and rental market in Lesotho. Thus, its equivalent rental value was estimated to that of a "typical" facility using the 2012 subsidized factory rental rates from the Lesotho National Development Corporation (IMF 2012).

- VEID unit cost: Lesotho is a lower middle income country while Rwanda is a low income country. Thus, the VEID unit cost from Lesotho may not necessarily be a full representation of VEID unit cost in Rwanda. 
Project SOAR

Population Council 4301 Connecticut Ave, NW,

Suite 280

Washington, D.C. 20008 USA

Tel: +1 2022379400

Fax: +1 2022378410

Ideas. Evidence. Impact.

projsoar.org 